TRANSACTIONS OF THE

AMERICAN MATHEMATICAL SOCIETY

Volume 353, Number 3, Pages 1251-1278

S 0002-9947(00)02716-

Article electronically published on November 8, 2000

\title{
THE COMBINATORICS OF BERNSTEIN FUNCTIONS
}

\author{
THOMAS J. HAINES
}

\begin{abstract}
A construction of Bernstein associates to each cocharacter of a split $p$-adic group an element in the center of the Iwahori-Hecke algebra, which we refer to as a Bernstein function. A recent conjecture of Kottwitz predicts that Bernstein functions play an important role in the theory of bad reduction of a certain class of Shimura varieties (parahoric type). It is therefore of interest to calculate the Bernstein functions explicitly in as many cases as possible, with a view towards testing Kottwitz' conjecture. In this paper we prove a characterization of the Bernstein function associated to a minuscule cocharacter (the case of interest for Shimura varieties). This is used to write down the Bernstein functions explicitly for some minuscule cocharacters of $G l_{n}$; one example can be used to verify Kottwitz' conjecture for a special class of Shimura varieties (the "Drinfeld case"). In addition, we prove some general facts concerning the support of Bernstein functions, and concerning an important set called the " $\mu$-admissible" set. These facts are compatible with a conjecture of Kottwitz and Rapoport on the shape of the special fiber of a Shimura variety with parahoric type bad reduction.
\end{abstract}

\section{INTRODUCTION AND STATEMENT OF MAIN RESULTS}

Let $\mathcal{H}$ denote the Iwahori-Hecke algebra of an almost simple, split connected reductive group $G$ over a $p$-adic field $F$. More concretely, if $I \subset G(F)$ is an Iwahori subgroup, then $\mathcal{H}$ is the convolution algebra of compactly supported $I$-bi-invariant functions on $G(F)$, where convolution is defined using the Haar measure on $G(F)$ which gives $I$ volume 1 . Let $q$ denote the size of the residue field of $F$, and let $\mathbb{Z}^{\prime}=\mathbb{Z}\left[q^{1 / 2}, q^{-1 / 2}\right]$. Fix an $F$-split maximal torus $T$ of $G$ and a Borel subgroup $B$ containing $T$. Let $W$ denote the Weyl group of $G$ and denote the cocharacter lattice by $X_{*}(T)$. Bernstein has constructed a $\mathbb{Z}^{\prime}$-algebra isomorphism between the Weyl group invariant elements of the group algebra of $X_{*}(T)$ and the center of $\mathcal{H}$ :

$$
\mathbb{Z}^{\prime}\left[X_{*}(T)\right]^{W} \stackrel{\sim}{\longrightarrow}(\mathcal{H}) .
$$

This is achieved by constructing, for each dominant cocharacter $\mu \in X_{*}(T)$, an element $z_{\mu}$ in the center of $\mathcal{H}$ (see $\S 2$ for the definition), and then by showing these elements form a $\mathbb{Z}^{\prime}$-basis for $Z(\mathcal{H})$, as $\mu$ runs over all dominant cocharacters. We call the elements $z_{\mu}$ the Bernstein functions.

The main aim of this paper is to study the Bernstein functions from a combinatorial viewpoint, meaning that we seek an explicit expression for $z_{\mu}$ as a $\mathbb{Z}^{\prime}$-linear combination of the standard generators $T_{w}$, where $T_{w}$ is the characteristic function of the Iwahori double coset corresponding to the element $w$ in the extended affine Weyl group $\widetilde{W}$ of $G$ (according to the Iwahori decomposition, $\widetilde{W}$ indexes the set

Received by the editors July 12, 1999.

2000 Mathematics Subject Classification. Primary 20C08; Secondary 14G35. 
$I \backslash G(F) / I$ ). A second goal of this article is the study of the $\mu$-admissible subset of the extended affine Weyl group (see $\S 2$ for definition).

The motivation for this work comes from two conjectures in the theory of Shimura varieties with parahoric type bad reduction, which we now briefly explain. Fix a rational prime $p$. A Shimura variety $S_{K}$ is, roughly speaking, attached to a triple of group-theoretic data $(G, K,\{\mu\})$, where $G$ is a connected reductive $\mathbb{Q}$-group, $K=K_{p} K^{p} \subset G\left(\mathbb{Q}_{p}\right) \times G\left(\mathbb{A}_{f}^{p}\right)$ is a compact open subgroup, and $\{\mu\}$ is the $G\left(\overline{\mathbb{Q}}_{p}\right)$ conjugacy class of a cocharacter $\mu:\left(\mathbb{G}_{m}\right)_{\overline{\mathbb{Q}}_{p}} \rightarrow G_{\overline{\mathbb{Q}}_{p}}$ which is minuscule, meaning that $\langle\alpha, \mu\rangle \in\{-1,0,1\}$, for every root $\alpha$ of $G$. It is known that $S_{K}$ is defined over a number field $E$. We say $S_{K}$ is of parahoric type (resp. Iwahori type) (at $p$ ) if $K_{p}$ is a parahoric (resp. Iwahori) subgroup of $G\left(\mathbb{Q}_{p}\right)$, meaning that the "reduction modulo $p$ " of $K_{p}$ is a parabolic (resp. Borel) subgroup of $G\left(\mathbb{F}_{p}\right)$.

The motivating conjectures concern the "semi-simplified" local zeta function $Z_{\mathfrak{p}}^{s s}\left(S_{K}, s\right)$ of a parahoric type Shimura variety $S_{K}$ at a prime ideal $\mathfrak{p}$ of $E$ dividing $p$. This is considered by M. Rapoport in [11. Assume that $E_{\mathfrak{p}}$ is an unramified extension of $\mathbb{Q}_{p}$. By reasoning that is analogous to the case of good reduction, Rapoport shows in loc. cit. that in order to understand $Z_{\mathfrak{p}}^{s s}\left(S_{K}, s\right)$ one should study the function on $S_{K}\left(\mathbb{F}_{q}\right)$ defined by

$$
x_{0} \mapsto \operatorname{tr}\left(F r_{q} ; R \Psi_{x_{0}}^{\mathcal{I}}\left(\overline{\mathbb{Q}}_{l}\right)\right),
$$

where $R \Psi$ is the sheaf of nearby cycles on $\left(S_{K}\right)_{\overline{\mathbb{F}}_{q}}, q=p^{j}$ is such that $\mathbb{Q}_{p^{j}}$ contains $E_{\mathfrak{p}}, x_{0} \in S_{K}\left(\mathbb{F}_{q}\right), F r_{q}$ is the geometric Frobenius on $\left(S_{K}\right)_{\mathbb{F}_{q}}$, and we take invariants under $\mathcal{I}$, the inertia subgroup of $\operatorname{Gal}\left(\overline{\mathbb{Q}}_{p} / \mathbb{Q}_{p}\right)$. We need a purely group-theoretic interpretation of this function, if we are eventually going to use the Arthur-Selberg trace formula to express the local zeta function in terms of automorphic $L$-functions.

Such an interpretation has been conjectured by R. Kottwitz. To state Kottwitz' conjecture, fix an unramified extension $F=\mathbb{Q}_{p^{j}} \supset E_{\mathfrak{p}}$, and assume that $G$ is split over $F$. For simplicity we also assume that $K_{p}$ is an Iwahori subgroup. Then there is an Iwahori subgroup $I \subset G(F)$ such that $I \cap G\left(\mathbb{Q}_{p}\right)=K_{p}$. Write $p^{j}=q$, and $\mathcal{H}=C_{c}(I \backslash G(F) / I)$. Associated to the element $\{\mu\}$ in the Shimura datum is the Bernstein function $z_{\mu} \in Z(\mathcal{H})$.

Locally in the étale topology, the special fiber of $S_{K}$ has a stratification indexed by certain elements of the extended affine Weyl group $\widetilde{W}$ of $G$. In fact, $S_{K}$ is étale locally isomorphic to a "local model" $M^{\text {loc }}$ (see [11]) and the special fiber of $M^{\text {loc }}$ is a set of Iwahori orbits in the affine flag variety for $G\left(\overline{\mathbb{F}}_{p}((t))\right)$. To $x_{0}$ we associate the element $x \in \widetilde{W}$ indexing the stratum containing $x_{0}$.

Conjecture 1 (Kottwitz). In the case of Iwahori type reduction,

$$
\operatorname{tr}\left(F r_{q} ; R \Psi_{x_{0}}^{\mathcal{I}}\left(\overline{\mathbb{Q}}_{l}\right)\right)=q^{\operatorname{dim}\left(S_{K}\right) / 2} z_{\mu}(x),
$$

where $q$ is the cardinality of the residue field of $F$, and $z_{\mu}(x)$ is the coefficient of $T_{x}$ for the Bernstein function $z_{\mu}$ in $C_{c}(I \backslash G(F) / I)$ corresponding to the cocharacter $\mu$.

Concerning the shape of the stratification of the "local model" we have the following conjecture.

Conjecture 2 (Kottwitz-Rapoport). The strata of $M^{\text {loc }}$ are indexed by elements of the $\mu$-admissible set.

If Conjecture 1 were true it would follow that $q^{\operatorname{dim}\left(S_{K}\right) / 2} z_{\mu}$ is the correct "test function" which is "plugged into" the twisted orbital integrals which come into the 
computation of the semi-simplified local zeta function when one attempts to use the Arthur-Selberg trace formula. This is in contrast to the case of good reduction, where $K_{p}$ is a hyperspecial maximal compact subgroup, and the test function is simply the characteristic function of the double coset $K_{p}(F) \mu\left(p^{-1}\right) K_{p}(F)$ in the spherical Hecke algebra $C_{c}\left(K_{p}(F) \backslash G(F) / K_{p}(F)\right.$ ) (see $\S 16$ of $[9]$ ).

In [1], Rapoport has given explicit formulae in the "Drinfeld case" for the trace of Frobenius on nearby cycles appearing in Conjecture 1 (see loc. cit. Thm 3.12). In order to compare these formulae with Kottwitz' group-theoretic prescription, one needs to compute explicitly the coefficients $z_{\mu}(x)$ of the Bernstein function $z_{\mu}$ with respect to the basis $\left\{T_{x}, \quad(x \in \widetilde{W})\right\}$ of $\mathcal{H}$.

The main theorem of this paper is a characterization of $z_{\mu}$ for any minuscule cocharacter, which is Theorem 5.8 in $\S 5$ :

Theorem 3 (Main Theorem). Let $\mu$ be a minuscule dominant cocharacter. Then $z_{\mu}$ is the unique element of $\mathcal{H}$ such that the following three properties hold:

(1) $z_{\mu} \in Z(\mathcal{H})$,

(2) $\operatorname{supp}\left(z_{\mu}\right)$ is contained in the $\mu$-admissible set,

(3) $z_{\mu}(\mu)=1$.

We use the characterization to compute the coefficients $z_{\mu}(x)$ for Bernstein functions in certain important cases. The main application is the computation of the function $z_{(1,0, \ldots, 0)}$ for the group $G l_{n}$ (Proposition. 7.1). This can be reformulated as follows (cf. notation in $\S 2$ ).

Proposition 4. For $x$ an element of the extended affine Weyl group of $G l_{n}$, let $z_{\mu}(x)$ denote the coefficient of $T_{x}$ in the expression for the element $z_{\mu}$. Then for $\mu=\left(1,0^{n-1}\right)$, we have

$$
q^{\operatorname{dim}\left(S_{K}\right) / 2} z_{\mu}(x)= \begin{cases}(1-q)^{l(\mu)-l(x)} & \text { if } x \text { is } \mu \text {-admissible, } \\ 0 & \text { if } x \text { is not } \mu \text {-admissible. }\end{cases}
$$

Here we have used $\operatorname{dim}\left(S_{K}\right)=l(\mu)$, where $l(x)$ denotes the length of an element $x \in \widetilde{W}$ (see $\S 2$ for precise definition). Putting this formula for $z_{\mu}(x)$ together with Rapoport's explicit formulae for the trace of Frobenius on nearby cycles ([11]), one can prove that Conjecture 1 holds in the "Drinfeld case" (see [5] for further details and another proof of Proposition 4). A related Shimura variety associated to the data $\left(G l_{4}, \mu=(1,1,0,0)\right)$ has been studied by U. Görtz. He computed the trace of Frobenius on the sheaf of nearby cycles and, comparing the result with the formulae for the coefficients $z_{(1,1,0,0)}(x)$ given in $\S 9$, he verified that Conjecture 1 also holds in this case.

If Conjectures 1 and 2 were both true, one would expect the support of $z_{\mu}$ to lie in the $\mu$-admissible set. This is in fact true even if $\mu$ is not minuscule (see Corollary $5.7)$ :

Proposition 5. For any dominant cocharacter $\mu$, the support of $z_{\mu}$ is contained in the $\mu$-admissible set.

This result is the main ingredient in the proof of Theorem 3 above. The proof is inspired by a result of Deodhar ([4]) and uses a generalization of one of the definitions appearing in loc. cit. 
It appears to be true that the support of $z_{\mu}$ is precisely the $\mu$-admissible set. This is true in the case of $G l_{2}$, as follows from the Corollary 10.4 of $\S 10$. Moreover, it also holds for any group if $\mu$ is minuscule (see [5]). We make the following conjecture:

Conjecture 6. For any dominant cocharacter $\mu$, $\operatorname{supp}\left(z_{\mu}\right)$ is the $\mu$-admissible set.

We now outline the contents of the paper. In $\S 2$ we define notation and recall some important definitions. In $\S \S 3-5$ we establish general facts which will be required for the applications in later sections. In particular, in $\S 3$ we give simple necessary and sufficient conditions for an arbitrary element in the Iwahori-Hecke algebra to be central. In $\S 4$ we study the relationship between conjugacy and $\mu$-admissibility. In $\S 5$ the support of $z_{\mu}$ is characterized, leading to the proof of Proposition 5 (Corollary 5.7) and Theorem 3 (Theorem 5.8) cited above.

The second half of the paper is devoted to applications and examples of the general facts proved in $\S \S 3-5$. In $\S 6$, some facts about $\mu$-admissible sets for $G l_{n}$ and $\mu$ minuscule are proved; these are used in the proof of Proposition 4 (Proposition 7.1) mentioned above, which is given in $\S 7$. In $\S 8$ the complexity of the $\mu$-admissible sets is illustrated by counting the $\mu$-admissible set for some minuscule cocharacters of $G l_{n}$ and $G S p_{2 n}$. In $\S 9$, further examples of Bernstein functions for minuscule cocharacters of $G l_{n}$ and $G S p_{2 n}$ are given, using the technical results from $\S 6$.

Finally, in $\S 10$, we present a proof that the Bernstein and Satake isomorphisms are compatible, using results of G. Lusztig (7]) and S. Kato ([8]). This implies that for any dominant $\mu$ there is a unique function $k_{\mu}$ in $Z(\mathcal{H})$ whose image in the spherical Hecke algebra has Satake transform $q^{l(\mu) / 2} \chi_{\mu}$, where $\chi_{\mu}$ denotes the character of the highest weight module attached to $\mu$. We calculate the function $k_{\mu}$ explicitly for any dominant cocharacter $\mu$ of the group $G l_{2}$ (Proposition 10.3), and then use this to compute all the Bernstein functions for $G l_{2}$ (Corollary 10.4).

\section{FURTHER MOTATION}

2.1. Affine Weyl group. We denote by $\Pi$ the set of simple roots, and $S$ the corresponding set of simple reflections in $W$. Let $\tilde{\alpha}$ denote the unique highest root, and let $s_{0}=\tilde{\tilde{\alpha}} s_{\tilde{\alpha}}=s_{(-\tilde{\alpha}, 1)}$ denote the affine reflection about the affine hyperplane $\left\{x \in X_{*}\left(T^{a d}\right) \otimes \mathbb{R} \mid\langle\tilde{\alpha}, x\rangle=1\right\}$, where $T^{a d}$ is the image of $T$ in the adjoint group $G^{a d}$. Let $S_{a}=S \cup\left\{s_{0}\right\}$. We denote by $T^{s c}$ the inverse image of $T$ under the map $G^{s c} \rightarrow G$; $T^{s c}$ is a maximal torus in the simply connected cover $G^{s c}$ of $G^{d e r}$. The affine Weyl group is by definition the group $W_{a}=X_{*}\left(T^{s c}\right) \rtimes W$. It is known that $\left(W_{a}, S_{a}\right)$ is a Coxeter group; therefore there is a length function $l(x)$ and a Bruhat order $\leq$ defined on $W_{a}$. If $\Omega$ denotes the subgroup of $\widetilde{W}$ which preserves the set $S_{a}$ under conjugation, then $\widetilde{W}=W_{a} \rtimes \Omega$. We extend the length function to $\widetilde{W}$ by declaring it to be trivial on $\omega$, and we extend the Bruhat order by defining $x_{1} \omega_{1} \leq x_{2} \omega_{2}$ if and only if $x_{1} \leq x_{2}$ and $\omega_{1}=\omega_{2}$.

Fix a dominant cocharacter $\mu$.

Definition 2.1. We say an element $x \in \widetilde{W}$ is $\mu$-admissible if $x \leq w(\mu)$ for some $w \in W$.

2.2. Hecke algebra. The braid group of $\widetilde{W}$ is the group generated by symbols $T_{w}$ for $w \in \widetilde{W}$ subject to the relation $T_{w w^{\prime}}=T_{w} T_{w^{\prime}}$ whenever $l\left(w w^{\prime}\right)=l(w)+l\left(w^{\prime}\right)$. The Hecke algebra $\mathcal{H}$ is by definition the quotient of the group algebra (over $\mathbb{Z}^{\prime}$ ) of 
the braid group of $\widetilde{W}$, by the two-sided ideal generated by the elements

$$
\left(T_{s}+1\right)\left(T_{s}-q\right)
$$

for $s \in S_{a}$. We continue to denote by $T_{w}$ the image in $\mathcal{H}$ of the element $T_{w}$ in the braid group. It is known that the elements $T_{w}$ form a $\mathbb{Z}^{\prime}$-basis for $\mathcal{H}$.

For $\psi=\sum_{x \in \widetilde{W}} a_{x} T_{x} \in \mathcal{H}$, we call $\operatorname{supp}(\psi)=\left\{x \in \widetilde{W} \mid a_{x} \neq 0\right\}$ the support of $\psi$. We occasionally denote the coefficient $a_{x}$ by $\psi(x)$.

For any $x \in X_{*}$, define an element $\Theta_{x}=q^{-\left(l\left(x_{1}\right)-l\left(x_{2}\right)\right) / 2} T_{x_{1}} T_{x_{2}}^{-1}$, where $x=$ $x_{1}-x_{2}$ and $x_{i} \in X_{d o m}$, for $i=1,2$. This element is independent of the choice of $x_{1}$ and $x_{2}$, and moreover the elements $\Theta_{x} T_{w}$ (for $x \in X_{*}$ and $w \in W$ ) form a $\mathbb{Z}^{\prime}$ basis for $\mathcal{H}$. We use these to define the Bernstein function attached to a dominant cocharacter $\mu$ :

Definition 2.2. $z_{\mu}=\sum_{\lambda} \Theta_{\lambda}$, where $\lambda$ ranges over the $W$-orbit of $\mu$.

The following theorem is due to Bernstein (see [7):

Theorem 2.3 (Bernstein). The center $Z(\mathcal{H})$ of the Hecke algebra is the free $\mathbb{Z}^{\prime}$ module generated by the elements $z_{\mu}$, where $\mu$ ranges over all dominant cocharacters of $A$.

(Note: In [7] this is only stated for $G$ a split semi-simple group, but it is easy to deduce the general statement for $G$ any split quasi-simple connected reductive group.)

For any $T_{w}$, we define a renormalization $\tilde{T}_{w}=q^{-l(w) / 2} T_{w}$. Define a new parameter $Q=q^{-1 / 2}-q^{1 / 2}$. Then the elements $\tilde{T}_{w}$ also form a basis for $\mathcal{H}$, and the usual relations can be written as

$$
\widetilde{T}_{s} \widetilde{T}_{w}= \begin{cases}\widetilde{T}_{s w}, & \text { if } l(s w)=l(w)+1, \\ -Q \widetilde{T}_{w}+\widetilde{T}_{s w}, & \text { if } l(s w)=l(w)-1,\end{cases}
$$

for $w \in \widetilde{W}$ and $s \in S_{a}$. There is also a right-handed version of this relation. Note, in particular, that $\tilde{T}_{s}^{-1}=\tilde{T}_{s}+Q$, for $s \in S_{a}$.

\section{Conditions on central elements}

The purpose of this section is to write down in a convenient form the conditions for an arbitrary element $\psi=\sum_{w} b_{w} \widetilde{T}_{w}$ to be in the center of $\mathcal{H}$. The results of this section are used later $(\S \S 6-7,9)$ to construct certain elements in the center of the Iwahori-Hecke algebra. We then show these are in fact equal to Bernstein functions by using the characterization of Bernstein functions given in Theorem 3 .

First note that for any $s \in S_{a}, \tau \in \Omega$, and $w \in \widetilde{W}$, we have

$$
\begin{gathered}
\widetilde{T}_{s} \widetilde{T}_{w}= \begin{cases}\widetilde{T}_{s w}, & \text { if } l(s w)=l(w)+1, \\
-Q \widetilde{T}_{w}+\widetilde{T}_{s w}, & \text { if } l(s w)=l(w)-1,\end{cases} \\
\widetilde{T}_{w} \widetilde{T}_{s}= \begin{cases}\widetilde{T}_{w s}, & \text { if } l(w s)=l(w)+1, \\
-Q \widetilde{T}_{w}+\widetilde{T}_{w s}, & \text { if } l(w s)=l(w)-1,\end{cases} \\
\widetilde{T}_{\tau} \widetilde{T}_{w}=\widetilde{T}_{\tau w}, \\
\widetilde{T}_{w} \widetilde{T}_{\tau}=\widetilde{T}_{w \tau} .
\end{gathered}
$$


It follows that we can write, for $s \in S_{a}$, and $\tau \in \Omega$,

$$
\begin{gathered}
\widetilde{T}_{s} \psi=\sum_{w: l(s w)=l(w)+1} b_{w} \widetilde{T}_{s w}+\sum_{w: l(s w)=l(w)-1} b_{w}\left(-Q \widetilde{T}_{w}+\widetilde{T}_{s w}\right), \\
\psi \widetilde{T}_{s}=\sum_{w: l(w s)=l(w)+1} b_{w} \widetilde{T}_{w s}+\sum_{w: l(w s)=l(w)-1} b_{w}\left(-Q \widetilde{T}_{w}+\widetilde{T}_{w s}\right), \\
\widetilde{T}_{\tau} \psi=\sum_{w} b_{w} \widetilde{T}_{\tau w}, \\
\psi \widetilde{T}_{\tau}=\sum_{w} b_{w} \widetilde{T}_{w \tau} .
\end{gathered}
$$

On equating the coefficients of $\widetilde{T}_{x}$ in the first two equations (and also in the second two equations), we deduce that $\psi$ is in the center of the Iwahori-Hecke algebra if and only if the following five conditions hold, for every $x \in \widetilde{W}, s \in S_{a}$, and $\tau \in \Omega$ :

- If $l(s x)=l(x)-1$ and $l(x s)=l(x)-1$, then $b_{s x}=b_{x s}$,

- If $l(s x)=l(x)-1$ and $l(x s)=l(x)+1$, then $b_{s x}-Q b_{x}=b_{x s}$,

- If $l(s x)=l(x)+1$ and $l(x s)=l(x)-1$, then $b_{s x}=b_{x s}-Q b_{x}$

- If $l(s x)=l(x)+1$ and $l(x s)=l(x)+1$, then $b_{s x}=b_{x s}$,

- $b_{\tau x}=b_{x \tau}$.

Replacing $x$ with $x s$, we see that the first and fourth conditions can be expressed as a single condition, and similarly the second and third conditions, in the presence of the first and fourth, can be expressed as a single condition. We have proved the following Lemma:

Lemma 3.1. The element $\psi$ is in the center of the Iwahori-Hecke algebra if and only if the following three conditions hold, for every $x \in \widetilde{W}, s \in S_{a}$ and $\tau \in \Omega$ :

(1) If $l(s x s)=l(x)$, then $b_{s x s}=b_{x}$,

(2) If $l(s x s)=l(x)-2$, then $b_{x}=b_{s x s}-Q b_{x s}$,

(3) $b_{\tau x \tau^{-1}}=b_{x}$.

\section{Conjugacy And $\mu$-ADmissibility}

In this section we will establish some general facts concerning $\mu$-admissibility and conjugacy classes. These facts play a role in the proof of the main computation (Proposition 4 in the introduction).

The first step is an easy lemma that applies to arbitrary Coxeter groups.

Lemma 4.1. Let $(W, S)$ be a Coxeter group. Let $w \in W$ and $s, t \in S$. Suppose that $l(s w t)=l(w)$ and $l(w t)=l(s w)$. Then $s w=w t$.

Proof. This can be found on p.148 of [6], but we repeat the proof for convenience. Write $w=s_{1}, \cdots s_{r}$ (reduced). There are two possibilities, either $l(s w)>l(w)$ or $l(s w)<l(w)$. Suppose first that $l(s w)>l(w)$. Then $l(s w t)=l(w)<l(s w)$, so the Exchange Condition of Coxeter groups applies to the pair $s w, t$, meaning that $s w=w^{\prime} t$, where either $w^{\prime}=s s_{1} \cdots \hat{s}_{i} \cdots s_{r}$ or $w^{\prime}=w$. The first alternative is impossible because it would imply that $w=s(s w)=s\left(w^{\prime} t\right)=s_{1} \cdots \hat{s}_{i} \cdots s_{r} t$, forcing $w t$ to be shorter than $w$, contrary to assumption. Therefore $w^{\prime}=w$ and so $s w=w t$. 
Now suppose that $l(s w)<l(w)$. The hypotheses of the first case are now satisfied with $s w$ in place of $w$, so we can conclude that $w=s w t$.

Remark. The lemma as stated applies to affine Weyl groups $\left(W_{a}, S_{a}\right)$, but it easy to see that it remains true even if we allow $w$ to be an element of an extended affine Weyl group $\widetilde{W}$.

We now recall three basic facts about the Bruhat order.

Lemma 4.2. Let $x, y$ be elements of a Coxeter group $(W, S)$. Suppose that $x^{-1} y$ is a reflection and $l(x)<l(y)$. Then $x \leq y$ in the Bruhat order.

Proof. This is Lemma 8.11 of [1].

Lemma 4.3. Let $x, y \in \widetilde{W}$, and let $s \in S_{a}$. Assume that $x \leq y$. Then

(1) either $s x \leq y$ or $s x \leq s y$, and

(2) either $x \leq s y$ or $s x \leq s y$, and

(3) either $x s \leq y$ or $x s \leq y s$, and

(4) either $x \leq y s$ or $x s \leq y s$.

Proof. The first two statements, for ordinary or affine Weyl groups, are proved in Lemma 2.5 of [1. It is easy to check that they remain valid for extended affine Weyl groups. The last two statements follow from the first two and the fact that, for any $x, y \in \widetilde{W}, x \leq y$ if and only if $x^{-1} \leq y^{-1}$.

Lemma 4.4. Let $x, y \in \widetilde{W}$ and let $x=s_{1} \cdots s_{q}$ be a reduced expression of $x$. Then $y \leq x$ if and only if there exists a strictly increasing sequence $i_{1}<i_{2}<\cdots i_{k}$ (possibly empty) of integers drawn from $\{1,2, \ldots, q\}$, such that $y=s_{i_{1}} \cdots s_{i_{k}}$.

Proof. This is Proposition 2.8 of [1].

For the next lemma let $\mu$ denote an arbitrary dominant cocharacter of the torus $T$, the maximal $F$-split torus in $G$ we fixed above. Let $x$ denote an element of the extended affine Weyl group $\widetilde{W}$, and $s \in S_{a}$ a simple reflection in $W_{a}$.

Lemma 4.5. If $l(s x s)=l(x)$, then $x$ and sxs are simultaneously $\mu$-admissible.

Proof. We must prove that if the lengths of $x$ and $s x s$ are the same and $x$ is $\mu$ admissible, then sxs is $\mu$-admissible. Consider the four elements $x, s x, x s$, and $s x s$. Since $x$ and sxs have the same length, there are only four possible configurations of these four elements in the Bruhat order. Two of them involve the cases where $l(s x)=l(x s)$ (which necessarily differs from $l(x)=l(s x s)$ by one). But in these two cases we know by Lemma 4.1 (and following Remark) that $x=s x s$, so we are done. In the remaining two cases, either $x s$ is the least upper bound and $s x$ is the greatest lower bound of the set $\{x, x s, s x, s x s\}$ (in the Bruhat order), or vice-versa. Suppose for instance that $x s$ is the least upper bound, so that $x \leq x s$ and $s x s \leq x s$. If $x s$ is $\mu$-admissible, then so is $s x s$ and we are done. So assume that $x s$ is not $\mu$-admissible. We can write $x \leq \mu^{\prime}$ for some $\mu^{\prime} \in W \cdot \mu$. By Lemma 4.3 (3), we know that either $x s \leq \mu^{\prime}$ or $x s \leq \mu^{\prime} s$. The first possibility does not occur (since $x s$ is not $\mu$-admissible), so the second one does. Applying Lemma 4.3 (2) to this relation we see that either $x s \leq s \mu^{\prime} s=s\left(\mu^{\prime}\right)$ or $s x s \leq s\left(\mu^{\prime}\right)$. Since $s\left(\mu^{\prime}\right) \in W \cdot \mu$, only the second case occurs and $s x s$ is $\mu$-admissible. The case where $s x$ is the least upper bound is similar. 
Corollary 4.6 (of Proof). Let $\mu^{\prime} \in W(\mu)$ and $s \in S_{a}$. If $x \leq \mu^{\prime}$ and either $s x$ or $x s$ is not $\mu$-admissible, then $s(x) \leq s\left(\mu^{\prime}\right)$.

\section{The SUPPort OF $z_{\mu}$ AND THE MAIN THEOREM}

In this section we prove the main theoretical result of the paper, Theorem 5.8 (Theorem 3 in the introduction), which is a characterization of $z_{\mu}$ for $\mu$ minuscule. This is the principal tool used to produce the explicit expressions for $z_{\mu}$ given later (we write down some explicit expressions, and use the main theorem to show that they are in fact the desired Bernstein functions). To start the proof we give a characterization of the support of the function $\Theta_{\lambda}$ for any $\lambda \in X_{*}$ (see Proposition 5.4). Using this we then show that the support of $\Theta_{\lambda}$ is contained in the set $\{x \in \widetilde{W} \mid x \leq \lambda\}$ (Proposition 5.5). An immediate consequence is that $\operatorname{supp}\left(z_{\mu}\right)$ is contained in the $\mu$-admissible subset of $\widetilde{W}$, for any dominant $\mu \in X_{*}$ (Corollary 5.7). We then use these observations about the supports of arbitrary Bernstein functions to prove Theorem 5.8.

Suppose $\lambda=\lambda_{1}-\lambda_{2}$, where $\lambda_{1}$ and $\lambda_{2}$ are both dominant. Write $\lambda_{i}=w_{i} \sigma_{i}$ $\left(w_{i} \in W_{a}\right.$ and $\left.\sigma_{i} \in \Omega\right)$ for $i=1,2$. Then $\Theta_{\lambda}=\tilde{T}_{w_{1} \sigma_{1}} \tilde{T}_{w_{2} \sigma_{2}}^{-1}=\tilde{T}_{w_{1}} \tilde{T}_{\tau\left(w_{2}\right)}^{-1} \tilde{T}_{\tau}$, where $\tau=\sigma_{1} \sigma_{2}^{-1}$. Note that for support questions it is enough to understand the first two factors, meaning that we have effectively replaced $\widetilde{W}$ with the Coxeter group $\left(W_{a}, S_{a}\right)$. In fact, the results of this section apply to any Coxeter group. Therefore fix a Coxeter group $(W, S)$ and consider the Hecke algebra $H_{W}$ with $\mathbb{Z}^{\prime}$-basis $\left\{\tilde{T}_{w}\right\}$ $(w \in W)$ and having relations

$$
\widetilde{T}_{w} \widetilde{T}_{s}= \begin{cases}\widetilde{T}_{w s}, & \text { if } l(w s)=l(w)+1, \\ -Q \widetilde{T}_{w}+\widetilde{T}_{w s}, & \text { if } l(w s)=l(w)-1,\end{cases}
$$

for $w \in W$ and $s \in S$. Fix elements $v$ and $w$ in $W$. Denote by $\Theta(v, w)$ the element $\tilde{T}_{v} \tilde{T}_{w^{-1}}^{-1}$. We want to understand the support of the function $\Theta(v, w)$. This is simplified by the following positivity statement (where $\mathbb{Z}_{+}$denotes the nonnegative integers):

Lemma 5.1. Write $\Theta(v, w)=\sum_{x \in W} a_{x}(Q) \tilde{T}_{x}$. Then $a_{x}(Q) \in \mathbb{Z}_{+}[Q]$ for every $x \in W$.

Proof. This is an easy induction on $l(w)$, using the identity $\tilde{T}_{s}^{-1}=\tilde{T}_{s}+Q$ and

$$
\tilde{T}_{y}\left(\tilde{T}_{s}+Q\right)= \begin{cases}Q \tilde{T}_{y}+\tilde{T}_{y s}, & \text { if } l(y s)=l(y)+1 \\ \tilde{T}_{y s}, & \text { if } l(y s)=l(y)-1 .\end{cases}
$$

Now we need to recall a definition from [4]. Fix a reduced expression $w=$ $s_{1} s_{2} \cdots s_{r}$.

Definition 5.2. We call a sequence $\left[\alpha_{0}, \alpha_{1}, \ldots, \alpha_{r}\right]$ a subexpression for $w$ (with respect to $\left.w=s_{1} s_{2} \cdots s_{r}\right)$ if $\alpha_{0}=1$ and $\alpha_{j} \in\left\{\alpha_{j-1}, \alpha_{j-1} s_{j}\right\}$ for every $j \geq 1$.

The following definition generalizes Deodhar's notion of distinguished subexpression (see loc. cit.).

Definition 5.3. Let $v$ and $w$ be arbitrary elements of $W$. Fix for $w$ a reduced expression $w=s_{1} \cdots s_{r}$. We call a sequence $\left[\sigma_{0}, \sigma_{1}, \ldots, \sigma_{r}\right] v$-distinguished (with respect to $\left.w=s_{1} s_{2} \cdots s_{r}\right)$ if 
(1) $\sigma_{j} \in\left\{\sigma_{j-1}, \sigma_{j-1} s_{j}\right\}$, for every $j \geq 1$,

(2) $\sigma_{j} \leq \sigma_{j-1} s_{j}$, for every $j \geq 1$,

(3) $\sigma_{0}=v$.

Note that this last definition is actually a generalization of the one introduced by Deodhar in 44: any distinguished subexpression of $w$ is $v$-distinguished for $v=1$.

Given $w=s_{1} s_{2} \cdots s_{r}$ as above and $v \in W$, define $D(v, w) \subset W$ to be the set of elements $\sigma_{r}$ such that there exists a subexpression $\left[\alpha_{0}, \ldots, \alpha_{r}\right]$ of $w$ such that $\left[v \alpha_{0}, \ldots, v \alpha_{r}\right]$ is $v$-distinguished and $v \alpha_{r}=\sigma_{r}$.

Proposition 5.4. $\operatorname{supp}(\Theta(v, w))=D(v, w)$.

Proof. The proof is by induction on $r$. If $r=0$ the statement is evident. Assume the result holds for $r \geq 0$; we will show it holds for $r+1$. Suppose therefore that we have a reduced expression $w s=s_{1} s_{2} \cdots s_{r} s$. Note that $\Theta(v, w s)=\Theta(v, w)\left(\tilde{T}_{s}+Q\right)$.

First we claim that $\operatorname{supp}(\Theta(v, w s)) \subset D(v, w s)$. Any element in the left-hand side must be in the support of $\tilde{T}_{\sigma_{r}}\left(\tilde{T}_{s}+Q\right)$ for some element $\sigma_{r} \in \operatorname{supp}(\Theta(v, w))$. We must consider two cases.

Case 1: $\sigma_{r} s<\sigma_{r}$. Then $\tilde{T}_{\sigma_{r}}\left(\tilde{T}_{s}+Q\right)=\tilde{T}_{\sigma_{r} s}$ and we must show $\sigma_{r} s \in D(v, w s)$. But by the induction hypothesis there is a subexpression $\left[\alpha_{0}, \ldots, \alpha_{r}\right]$ of $w$ such that $\left[v \alpha_{0}, \ldots, v \alpha_{r}\right]$ is distinguished, with $\sigma_{r}=v \alpha_{r}$. But then it is clear that $\left[\alpha_{0}, \ldots, \alpha_{r}, \alpha_{r} s\right]$ is a subexpression of $w s$ and $\left[v \alpha_{0}, \ldots, v \alpha_{r}, v \alpha_{r} s\right]$ is distinguished and $\sigma_{r} s=v \alpha_{r} s$, implying that $\sigma_{r} s \in D(v, w s)$.

Case 2: $\sigma_{r}<\sigma_{r} s$. Then $\tilde{T}_{\sigma_{r}}\left(\tilde{T}_{s}+Q\right)=Q \tilde{T}_{\sigma_{r}}+\tilde{T}_{\sigma_{r} s}$ and we have to show that $\sigma_{r} s$ and $\sigma_{r}$ are in $D(v, w s)$. The same argument as above shows that $\sigma_{r} s \in D(v, w s)$. The induction hypothesis implies the existence of a subexpression $\left[\alpha_{0}, \ldots, \alpha_{r}\right]$ of $w$ such that $\left[v \alpha_{0}, \ldots, v \alpha_{r}\right]$ is distinguished and $v \alpha_{r}=\sigma_{r}$. Note that $\left[\alpha_{0}, \ldots, \alpha_{r}, \alpha_{r}\right]$ is a subexpression for $w s$ and $\left[v \alpha_{0}, \ldots, v \alpha_{r}, v \alpha_{r}\right]$ is distinguished. This shows that $\sigma_{r} \in D(v, w s)$, and proves the claim.

Now we claim that $\operatorname{supp}(\Theta(v, w s)) \supset D(v, w s)$. Suppose $\left[\alpha_{0}, \ldots, \alpha_{r+1}\right]$ is a subexpression of $w s$ and $\left[v \alpha_{0}, \ldots, v \alpha_{r+1}\right]$ is distinguished. Write $\sigma_{j}=v \alpha_{j}$ for $j \geq 0$. We must show $\sigma_{r+1} \in \operatorname{supp}(\Theta(v, w s))$. By induction we know that $\sigma_{r} \in$ $\operatorname{supp}(\Theta(v, w))$.

Case 1: $\sigma_{r+1}=\sigma_{r}$. Then since $\left[\sigma_{0}, \ldots, \sigma_{r}, \sigma_{r}\right]$ is distinguished, we have $\sigma_{r}<\sigma_{r} s$ and so $\tilde{T}_{\sigma_{r}}\left(\tilde{T}_{s}+Q\right)=Q \tilde{T}_{\sigma_{r}}+\tilde{T}_{\sigma_{r} s}$. It follows that $\sigma_{r+1}=\sigma_{r} \in \operatorname{supp}(\Theta(v, w s))$.

Case 2: $\sigma_{r+1}=\sigma_{r} s$. If $\sigma_{r}<\sigma_{r} s$, then $\tilde{T}_{\sigma_{r}}\left(\tilde{T}_{s}+Q\right)=Q \tilde{T}_{\sigma_{r}}+\tilde{T}_{\sigma_{r} s}$ and so $\sigma_{r+1}=\sigma_{r} s \in \operatorname{supp}(\Theta(v, w s))$. If $\sigma_{r} s<\sigma_{r}$, then $\tilde{T}_{\sigma_{r}}\left(\tilde{T}_{s}+Q\right)=\tilde{T}_{\sigma_{r} s}$ and so $\sigma_{r+1}=\sigma_{r} s \in \operatorname{supp}(\Theta(v, w s))$. This completes the proof.

Proposition 5.5. Suppose $\left[\alpha_{0}, \ldots, \alpha_{r}\right]$ is a subexpression of $w=s_{1} \cdots s_{r}$ and $\left[v, v \alpha_{1}, \ldots, v \alpha_{r}\right]$ is $v$-distinguished. Then $v \alpha_{r} \leq v w$.

Proof. We use induction on $r$. If $r=0$ the statement is evident. We assume the statement holds for $r \geq 0$ and deduce it for $r+1$. Suppose therefore that $w s_{r+1}=$ $s_{1} \cdots s_{r} s_{r+1}$ is a reduced expression, that $\left[\alpha_{0}, \ldots, \alpha_{r+1}\right]$ is a subexpression of $w s_{r+1}$ and $\left[v, v \alpha_{1}, \ldots, v \alpha_{r+1}\right]$ is $v$-distinguished with respect to $w s_{r+1}=s_{1} \cdots s_{r} s_{r+1}$. We want to prove that $v \alpha_{r+1} \leq v w s_{r+1}$. By Lemma 4.4 we can write $\alpha_{r+1}=$ $s_{1} \cdots \hat{s}_{i_{1}} \cdots \hat{s}_{i_{q}} \cdots s_{r+1}$, for $1 \leq i_{1}<\cdots<i_{q} \leq r+1$ and where the ' means we omit that term.

If $q=0$, then $v \alpha_{r+1}=v w s_{r+1}$ and so we are done. Therefore, we can assume $q>0$. We consider two cases. 
Case 1: $\alpha_{1}=s_{1}$. This means that $i_{1}>1$ and our sequence is of the form

$$
\left[v, v s_{1}, \ldots, v \alpha_{r+1}\right] .
$$

The last $r+1$ terms of this sequence form a $v s_{1}$-distinguished sequence with respect to $s_{2} \cdots s_{r+1}$. Therefore by the induction hypothesis we have $v s_{1}\left(s_{2} \cdots \hat{s}_{i_{1}} \cdots \hat{s}_{i_{q}}\right.$ $\left.\cdots s_{r+1}\right) \leq v s_{1}\left(s_{2} \cdots s_{r+1}\right)$, i.e., $v \alpha_{r+1} \leq v w s_{r+1}$.

Case 2: $\alpha_{1}=\alpha_{0}=1$. Then $i_{1}=1$ and our sequence is of the form

$$
\left[v, v, \ldots, v \alpha_{r+1}\right] \text {. }
$$

The last $r+1$ terms of this sequence form a $v$-distinguished sequence with respect to $s_{2} \cdots s_{r+1}$ and so by the induction hypothesis we have $v \alpha_{r+1} \leq v\left(s_{2} \cdots s_{r+1}\right)$. Since the sequence is distinguished, we have $v<v s_{1}$ and so the desired conclusion follows from the following lemma (taking $n=r+1$ ).

Lemma 5.6. Let $s_{1} \cdots s_{n}$ be reduced. Suppose $v<v s_{1}$. Then $v s_{2} \cdots s_{n}<v s_{1} \cdots s_{n}$.

Proof. Use induction on $n$. If $n=1$, then the statement is evident. Suppose that it holds for $n \geq 1$; we show it holds for $n+1$. We assume $v<v s_{1}$. By the induction hypothesis we have $v s_{2} \cdots s_{n}<v s_{1} \cdots s_{n}$. We want to show $v s_{2} \cdots s_{n+1}<v s_{1} \cdots s_{n+1}$. Call the first term $x$ and the second $y$. Note that $x^{-1} y=s_{n+1} \cdots s_{2} s_{1} s_{2} \cdots s_{n+1}$ is a reflection. By Lemma 4.2, we need only show $l(x)<l(y)$. Assume this is not the case. Then because $x^{-1} y$ is a reflection, it is impossible to have $l(x)=l(y)$. Therefore $l(y)<l(x)$ and hence $x \not \leq y$. The induction hypothesis asserts that $x s_{n+1}<y s_{n+1}$, so by Lemma 4.3 we deduce $x \leq y s_{n+1}$ and $x s_{n+1} \leq y$. Equality is impossible in each of these cases, for otherwise $s_{1} \cdots s_{n+1}=s_{2} \cdots s_{n}$. Hence we have strict inequalities

$$
l\left(x s_{n+1}\right)<l(y)<l(x),
$$

which is a contradiction because the numbers on the left and right must differ by 1. Therefore we conclude that $l(x)<l(y)$ and so $x \leq y$.

Corollary 5.7. Let $\lambda \in X_{*}$ and $\mu \in X_{d o m}$. Then the following statements hold:

(1) The support of $\Theta_{\lambda}$ is contained in $\{x \mid x \leq \lambda\}$.

(2) The support of $z_{\mu}$ is contained in the $\mu$-admissible set.

Proof. Using the notation at the beginning of this section we see that $\lambda=\lambda_{1} \lambda_{2}^{-1}=$ $w_{1}^{\tau}\left(w_{2}\right)^{-1} \tau$, where $\tau=\sigma_{1} \sigma_{2}^{-1}$ and $\Theta_{\lambda}=\tilde{T}_{w_{1}} \tilde{T}_{\tau\left(w_{2}\right)}^{-1} \tilde{T}_{\tau}$. Therefore we need to show that $\operatorname{supp}\left(\Theta\left(w_{1},{ }^{\tau}\left(w_{2}^{-1}\right)\right)\right.$ is contained in the set $\left\{x \mid x \leq w_{1}{ }^{\tau}\left(w_{2}^{-1}\right)\right\}$. This follows immediately from the previous two propositions. Thus the first statement is proved, and the second is a direct consequence of the first and the definition of $z_{\mu}$.

Now we can prove the main theorem.

Theorem 5.8. Let $\mu$ be a minuscule dominant cocharacter. Then $z_{\mu}$ is the unique element of $\mathcal{H}$ such that the following three properties hold:

(1) $z_{\mu} \in Z(\mathcal{H})$,

(2) $\operatorname{supp}\left(z_{\mu}\right)$ is contained in the $\mu$-admissible set,

(3) $z_{\mu}(\mu)=1$. 
Proof. It is obvious that $z_{\mu}$ satisfies conditions (1) and (3). Corollary 5.7 above shows it also satisfies condition (2).

We now want to prove that if $f$ satisfies these three conditions, then it must be $z_{\mu}$. First we need a lemma.

Lemma 5.9. Suppose $\lambda \in X_{\text {dom }}$ is $\mu$-admissible. Then $\lambda=\mu$.

Proof. For any cocharacter $x$ let $w_{x}$ denote the unique element of greatest length in the double coset $W x W$. It is well known that for dominant cocharacters $\lambda$ and $\mu$ we have

$$
w_{\lambda} \leq w_{\mu} \text { if and only if } \lambda \preceq \mu,
$$

where $\lambda \preceq \mu$ means $\mu-\lambda$ is 0 or a sum of simple coroots. Now $\lambda$ being $\mu$-admissible implies $\lambda \leq w \mu w^{-1}$ for some $w \in W$. It follows easily (using Lemma 4.3 repeatedly) that $w_{\lambda} \leq w_{\mu}$ and thus $\lambda \preceq \mu$. Since $\mu$ (being minuscule) is a minimal nonzero element of $X_{d o m}$ with respect to $\preceq$, we conclude that $\lambda=\mu$.

Now suppose $f \in \mathcal{H}$ satisfies the three conditions. Because $f \in Z(\mathcal{H})$, Bernstein's theorem (Theorem 2.3) implies that we can write $f$ as a $\mathbb{Z}^{\prime}$-linear combination of elements $z_{\lambda}$, for $\lambda \in X_{d o m}: f=\sum_{\lambda} a_{\lambda} z_{\lambda}$. Among those $\lambda$ such that $a_{\lambda} \neq 0$, choose one of maximal length; say $l(\lambda)=r$. Then we can write

$$
f=\sum_{\lambda^{\prime}: l\left(\lambda^{\prime}\right)=r} a_{\lambda^{\prime}} z_{\lambda^{\prime}}+\sum_{\lambda^{\prime \prime}: l\left(\lambda^{\prime \prime}\right)<r} a_{\lambda^{\prime \prime}} z_{\lambda^{\prime \prime}}
$$

Since $\operatorname{supp}\left(z_{\lambda^{\prime \prime}}\right)$ is contained in the $\lambda^{\prime \prime}$-admissible set for each $\lambda^{\prime \prime}$ and similarly for each $\lambda^{\prime}$ (Cor. 5.7), and the $W$-orbits of the elements $\lambda^{\prime}$ are disjoint, it follows that $\lambda \in \operatorname{supp}(f)$. By (2), this means $\lambda$ is $\mu$-admissible. Hence by the lemma above, $\lambda=\mu$. Since $\lambda$ was chosen arbitrarily (among the $\lambda^{\prime}$ 's), we can now write

$$
f=a_{\mu} z_{\mu}+\sum_{\lambda^{\prime \prime}: l\left(\lambda^{\prime \prime}\right)<r} a_{\lambda^{\prime \prime}} z_{\lambda^{\prime \prime}}
$$

Suppose that the sum on the right-hand side is nonempty. Then among the elements $\lambda^{\prime \prime}$ such that $a_{\lambda^{\prime \prime}} \neq 0$, choose one of maximal length, say $\lambda_{1}$. Then again by the argument used above we see that $\lambda_{1} \in \operatorname{supp}\left(\sum_{\lambda^{\prime \prime}} a_{\lambda^{\prime \prime}} z_{\lambda^{\prime \prime}}\right)$. We now reach a contradiction: If $\lambda_{1} \in \operatorname{supp}(f)$, then by (2) and the lemma above, $\lambda_{1}=\mu$, which can't happen (consider lengths). On the other hand, if $\lambda_{1} \notin \operatorname{supp}(f)$, then we must have $\lambda_{1} \in \operatorname{supp}\left(z_{\mu}\right)$, which again by the lemma leads to $\lambda_{1}=\mu$. This contradiction shows that the sum is in fact empty, so that

$$
f=a_{\mu} z_{\mu}
$$

Finally condition (3) implies that $a_{\mu}=1$.

\section{Applications to $G l_{n}$}

The goal of this section is to extend some results in [10] and then use these new results together with Theorem 5.8 to give an explicit formula for the Bernstein function attached to $G l_{n}$ and $\mu=(1,0, \ldots, 0)$. Some further examples for $G l_{n}$ will also be discussed. 
6.1. Minuscule alcoves for $G l_{n}$. We will need some results and definitions pertaining to the case $G=G l_{n}$. All the terminology and notation, and some of the theorems, will be taken from [10].

The extended affine Weyl group $\widetilde{W}$ for $G l_{n}$ is the semidirect product of the symmetric group $S_{n}$ and the group $\mathbb{Z}^{n}$. We view $\widetilde{W}$ as a group of affine linear transformations of $\mathbb{R}^{n}$, with $S_{n}$ acting by permutations of the coordinates and $\mathbb{Z}^{n}$ acting by translations. We order the affine roots in such a way that the simple affine roots are the functions $\left(x_{1}, \ldots, x_{n}\right) \mapsto x_{i}-x_{i+1}(1 \leq i \leq n-1)$ together with the affine linear function $\left(x_{1}, \ldots, x_{n}\right) \mapsto x_{n}-x_{1}+1$. The corresponding simple reflections $S_{a}$ are the transpositions $s_{i}=(i, i+1)$ in $S_{n}$ together with the affine linear tranformation $s_{0}$ given by $\left(x_{1}, \ldots, x_{n}\right) \mapsto\left(x_{n}+1, x_{2}, x_{3}, \ldots, x_{n-2}, x_{n-1}\right.$, $\left.x_{1}-1\right)$. The affine Weyl group $W_{a}$ is the semidirect product of $S_{n}$ with the subgroup of $\mathbb{Z}^{n}$ consisting of elements $\left(x_{1}, \ldots, x_{n}\right) \in \mathbb{Z}^{n}$ such that $x_{1}+\cdots+x_{n}=0$. We define the length function $l(\cdot)$ and the Bruhat order on the groups $W_{a}$ and $\widetilde{W}$ using the simple affine reflections $S_{a}$. (Note that these are the usual simple reflections defined by using the maximal torus $T$ of diagonal matrices and the usual Borel subgroup $B$ of upper triangular matrices.)

For a vector $v \in \mathbb{Z}^{n}$ we denote by $v(m)$ the $m$-th entry of $v$; thus $v=(v(1), \ldots$, $v(n))$. Also we write $\sum(v)$ for the $\operatorname{sum} v(1)+\cdots+v(n)$. Given two vectors $u, v \in \mathbb{Z}^{n}$, we write $u \leq v$ if $u(m) \leq v(m)$ for all $m$ such that $1 \leq m \leq n$.

Definition 6.1. An alcove for $G l_{n}$ is a sequence $\mathbf{v}$ consisting of vectors $v_{0}, v_{1}, \ldots$, $v_{n-1}, v_{n}=v_{0}+(1, \ldots, 1)$ in $\mathbb{Z}^{n}$ satisfying the following two conditions:

- $v_{0} \leq v_{1} \leq \cdots \leq v_{n-1} \leq v_{n}$,

- $\sum\left(v_{i}\right)=\sum\left(v_{i-1}\right)+1$, for $1 \leq i \leq n$.

We will call a vector $v=(v(1), \ldots, v(n)) \in \mathbb{Z}^{n}$ minuscule if $0 \leq v(m) \leq 1$ for all $m$ such that $1 \leq m \leq n$.

For each $r$ such that $0 \leq r \leq n$, let $\mathbf{e}_{r}$ denote the $r$-th standard basis vector in $\mathbb{R}^{n}$, with the convention $\mathbf{e}_{0}=(0, \ldots, 0)$. Also we will let $\omega_{r}$ denote the minuscule vector $\left(1^{r}, 0^{n-r}\right)$.

Definition 6.2. We say that an alcove $\mathbf{v}$ consisting of the vectors $v_{0}, \ldots v_{n}$ is minuscule if

$$
\omega_{i} \leq v_{i} \leq \omega_{i}+(1, \ldots, 1)
$$

for all $i$ in the range $0 \leq i \leq n-1$.

We refer to the minuscule alcove $\omega_{0}, \ldots, \omega_{n}$ as the standard alcove. The group $\widetilde{W}$ acts on the set of alcoves in the obvious way. It is easy to see that this action is simply transitive. Therefore by taking the standard alcove as base point, we may identify $\widetilde{W}$ with the set of all alcoves. If $\mathbf{v}$ is an alcove and $x$ is the corresponding element of $\widetilde{W}$, we write $\mathbf{v} \longleftrightarrow x$. If $w \in \widetilde{W}$ and $w \mathbf{v}$ is the alcove attained by the action of $w$ on $\mathbf{v}$, then we have $w \mathbf{v} \longleftrightarrow w x$.

Definition 6.3. We call an element $x \in \widetilde{W}$ minuscule if $r(0 \leq r \leq n)$ exists such that $x \leq v$ in the Bruhat order on $\widetilde{W}$, for some permutation $v$ of $\omega_{r}$.

In other words, $x$ is minuscule if and only if it is $\mu$-admissible for some minuscule dominant cocharacter $\mu$ (all of which are of the form $\omega_{r}$ for some $r$ such that $0 \leq r \leq n)$. For the rest of this paper we will tacitly assume the following theorem. 
Theorem 6.4 (Kottwitz-Rapoport). Suppose the alcove $\mathbf{v}$ corresponds to $x \in \widetilde{W}$. Then $\mathbf{v}$ is minuscule if and only if $x$ is minuscule.

Proof. This is a direct consequence of Theorem 3.5 of [10].

If $v_{0}, \ldots, v_{n}$ is a minuscule alcove, we associate to it a sequence of minuscule vectors $\mu_{0}, \ldots, \mu_{n-1}$, where

$$
\mu_{k}(j)=v_{k}(j)-\omega_{k}(j) .
$$

Also we define, for $1 \leq j \leq n$, the subset $K_{j}$ of $\{0,1,2, \ldots, n-1\}$ by putting

$$
K_{j}=\left\{k \mid 0 \leq k \leq n-1 \text { and } \mu_{k}(j)=1\right\} .
$$

Note that the set of vectors $\left\{\mu_{0}, \ldots, \mu_{n-1}\right\}$, or equivalently, the collection of sets $\left\{K_{1}, \ldots, K_{n}\right\}$, determines the minuscule alcove $v_{0}, \ldots, v_{n-1}$ uniquely.

Definition 6.5. For any alcove $\mathbf{v}$ and any integer $l$ in the range $0 \leq l \leq n-1$, we call the number $\mu_{l}(1)+\cdots+\mu_{l}(n)$ (which is independent of $l$ ) the size of $\mathbf{v}$.

Let $1 \leq i<j \leq n$ and $d \in \mathbb{Z}$. We will consider the decomposition of the affine reflection $w=w_{i, j ; d}=d\left(\mathbf{e}_{i}-\mathbf{e}_{j}\right) s$ corresponding to the affine linear function $\alpha_{i, j ; d}=x_{i}-x_{j}-d$ on $\mathbb{R}^{n}$. In our expression for $w$ here the term $d\left(\mathbf{e}_{i}-\mathbf{e}_{j}\right)$ denotes translation by this vector, and the term $s$ is the factor in the finite Weyl group; it is the transposition $(i j)$. Now suppose $\mathbf{v}$ is the alcove $v_{0}, \ldots, v_{n-1}$ and $x$ is the corresponding element of the extended affine Weyl group. Then we have a similar decomposition of $x$ :

$$
x=\left(v_{0}(1), \ldots, v_{0}(n)\right) \pi,
$$

where the first term is simply translation by the vector $v_{0} \in \mathbb{Z}^{n}$ and the second term is a permutation $\pi \in W$. We can also describe the alcove $\mathbf{v}$ by specifying the initial vector $v_{0}$ and the ordered set of vectors $\left(v_{1}-v_{0}, v_{2}-v_{1}, \ldots, v_{n}-v_{n-1}\right)$, which can also be written as $\left(\mathbf{e}_{\pi(1)}, \ldots, \mathbf{e}_{\pi(n)}\right)$.

If $K \subset \mathbb{Z} / n \mathbb{Z}$, we denote the complement of $K$ in $\mathbb{Z} / n \mathbb{Z}$ by $K^{c}$. The characteristic function of such a subset will be denoted by $\xi_{K}$.

The following are some facts which will be needed later.

Lemma 6.6 (Kottwitz-Rapoport). Let $\mathbf{v}$ be a minuscule alcove, and let $w$ be as above.

(1) For each $1 \leq j \leq n, K_{j}$ is either $\mathbb{Z} / n \mathbb{Z}, \emptyset$, or an interval of the form [?, $j$ ).

(2) Suppose that $w \mathbf{v}$ is minuscule. Then $d \in\{0,1\}$.

(3) If $d=0$, then $w \mathbf{v}$ is minuscule if and only if $[i, j) \subset K_{i}^{c} \cap K_{j}$.

(4) If $d=1$, then $w \mathbf{v}$ is minuscule if and only if $[i, j)^{c} \subset K_{j}^{c} \cap K_{i}$.

(5) Consider the statement: Either $d=0$ and $[i, j)=K_{i}^{c} \cap K_{j}$ or else $d=1$ and $[i, j)^{c}=K_{j}^{c} \cap K_{i}$. Then this statement holds if and only if $\mathbf{v} \leq w \mathbf{v}$ and $w \mathbf{v}$ is minuscule.

Proof. These are contained in $\S 5$ of [10].

Now fix $x, \mathbf{v}$, and $w$ as before. We denote by $\mathbf{v}^{\prime}, \mathbf{v}^{\prime \prime}$, and $\mathbf{v}^{\prime \prime \prime}$, the alcoves corresponding to the group elements $x w, w x$, and $w x w$, respectively. If these alcoves are minuscule, we also denote the corresponding collections of sets with the appropriate number of primes, that is, $\mathbf{v}^{\prime}$ corresponds to the collection of sets 
$\left\{K_{j}^{\prime}\right\}$, etc. We will follow the same convention when discussing the sets of minuscule vectors $\mu_{k}$, that is, $\mathbf{v}^{\prime}$ will correspond to the set $\left\{\mu_{j}^{\prime}\right\}$, etc. It is easy to see that

$$
\begin{aligned}
x & =v_{0} \pi, \\
x w & =\left(v_{0}+d\left(\mathbf{e}_{\pi(i)}-\mathbf{e}_{\pi(j)}\right)\right) \pi s, \\
w x & =\left(s\left(v_{0}\right)+d\left(\mathbf{e}_{i}-\mathbf{e}_{j}\right)\right) s \pi, \\
w x w & =\left(s\left(v_{0}\right)+d\left(\mathbf{e}_{i}-\mathbf{e}_{j}\right)+d\left(\mathbf{e}_{s \pi(i)}-\mathbf{e}_{s \pi(j)}\right)\right) s \pi s .
\end{aligned}
$$

If we consider the case where $d=0$, the expressions above can be simplified somewhat. In particular, we can write $w=s=(i j)$. In this case the associated alcoves may be specified by the following data:

$$
\begin{aligned}
& \mathbf{v} \longleftrightarrow\left(v_{0}(1), \ldots, v_{0}(n)\right) ; \mathbf{e}_{\pi(1)}, \ldots, \mathbf{e}_{\pi(n)}, \\
& \mathbf{v}^{\prime} \longleftrightarrow\left(v_{0}(1), \ldots, v_{0}(n)\right) ; \mathbf{e}_{\pi s(1)}, \ldots, \mathbf{e}_{\pi s(s)}, \\
& \mathbf{v}^{\prime \prime} \longleftrightarrow\left(v_{0}(s(1)), \ldots, v_{0}(s(n))\right) ; \mathbf{e}_{s \pi(1)}, \ldots \mathbf{e}_{s \pi(n)}, \\
& \mathbf{v}^{\prime \prime \prime} \longleftrightarrow\left(v_{0}(s(1)), \ldots, v_{0}(s(n))\right) ; \mathbf{e}_{s \pi s(1)}, \ldots \mathbf{e}_{s \pi s(n)} .
\end{aligned}
$$

Next we want to describe the conditions on the element $x=v_{0} \pi$ that ensure that $\mathbf{v}$ is a minuscule alcove:

Lemma 6.7. $\mathbf{v}$ is a minuscule alcove if and only if $v_{0}$ is a minuscule vector and the following condition holds, for every $1 \leq i \leq n$ :

$$
v_{0}(i)=0 \Rightarrow \pi^{-1}(i) \leq i, \text { and } v_{0}(i)=1 \Rightarrow \pi^{-1}(i) \geq i .
$$

Proof. This is an easy exercise using the definitions.

For $x$ and $w$ as above, define elements $i^{\prime}$ and $j^{\prime}$ such that $1 \leq i^{\prime}<j^{\prime} \leq n$ and $\pi\left(\left\{i^{\prime}, j^{\prime}\right\}\right)=\{i, j\}$. As an application of the previous lemma and the explicit expressions above we get the following:

Lemma 6.8. Suppose $x, x w, w x$, and wxw are all minuscule elements of $\widetilde{W}\left(G l_{n}\right)$. Then $\left\{i^{\prime}, j^{\prime}\right\} \cap\{i, j\}=\emptyset$.

Proof. We know from Lemma $6.6(2)$ that $d \in\{0,1\}$. First we consider the case $d=1$. In this case we will prove something stronger: if the translation parts of the four elements $x, x w, w x$, and $w x w$ are minuscule vectors, then $\left\{i^{\prime}, j^{\prime}\right\} \cap\{i, j\}=\emptyset$. Indeed, if the translation parts of the first and second elements are minuscule, we must have $v_{0}(\pi(i))=0$ and $v_{0}(\pi(j))=1$. On the other hand, if the translation parts of the first and third elements are minuscule, then $s\left(v_{0}\right)(i)=0$ and $s\left(v_{0}\right)(j)=1$, so $v_{0}(j)=0$ and $v_{0}(i)=1$. This implies that $\pi^{-1}(i) \neq i$ and $\pi^{-1}(j) \neq j$. Finally, if the translation parts of the first and fourth elements are minuscule, then we have $i \neq s \pi(i)$ and $j \neq s \pi(j)$, that is, $\pi^{-1}(j) \neq i$ and $\pi^{-1}(i) \neq j$. This proves the lemma in the case $d=1$.

Now consider the case $d=0$. Let $\tau=(1,0, \ldots, 0) c \in \Omega \subset \widetilde{W}\left(G l_{n}\right)$, where $c=(12 \cdots n) \in S_{n}=W\left(G l_{n}\right)$. Conjugating $w$ by an appropriate power of $\tau$ yields an element $w_{l, k ; 1}$. Indeed, a direct computation shows that for any $1 \leq i<j<n$ we have

$$
\begin{aligned}
\operatorname{Int}(\tau)\left(w_{i, j ; 0}\right) & =w_{i+1, j+1 ; 0}, \\
\operatorname{Int}(\tau)\left(w_{i, n ; 0}\right) & =w_{1, i+1 ; 1} .
\end{aligned}
$$

Moreover, conjugating any element of $\widetilde{W}\left(G l_{n}\right)$ by $\tau$ preserves the property of being minuscule, since $\operatorname{Int}(\tau)$ preserves the Bruhat order and the $W$-orbit of any minuscule 
dominant cocharacter $\mu$. Therefore we reduce this case to the case $d=1$ if we show that the cardinality of the set $\left\{i^{\prime}, j^{\prime}\right\} \cap\{i, j\}$ does not change when $x$ and $w$ are replaced with ${ }^{\tau} x$ and ${ }^{\tau} w$. The permutation factor of ${ }^{\tau} w$ is ${ }^{c} s=(i+1, j+1)$. The permutation factor of ${ }^{\tau} x$ is ${ }^{c} \pi$. Define $i^{\prime \prime}<j^{\prime \prime}$ by ${ }^{c} \pi\left(\left\{i^{\prime \prime}, j^{\prime \prime}\right\}\right)=\{i+1, j+1\}$. Then we must show that $\left|\left\{i^{\prime \prime}, j^{\prime \prime}\right\} \cap\{i+1, j+1\}\right|=\left|\left\{i^{\prime}, j^{\prime}\right\} \cap\{i, j\}\right|$. But it is clear from the definition that $\left\{i^{\prime \prime}, j^{\prime \prime}\right\}=\left\{i^{\prime}+1, j^{\prime}+1\right\}$, so the result follows.

\subsection{A technical lemma.}

Lemma 6.9. Suppose $w=w_{i, j ; d}$ is any affine reflection for $G l_{n}$ and $x$ is an element of $\widetilde{W}$. Suppose that $x, x w, w x$ and $w x w$ are all minuscule. Then the following statements hold:

(1) $K_{i}=K_{i}^{\prime}=\left[\pi^{-1}(i), i\right) \quad$ and $\quad K_{i}^{\prime \prime}=K_{i}^{\prime \prime \prime}=\left[\pi^{-1}(j), i\right)$,

(2) $K_{j}=K_{j}^{\prime}=\left[\pi^{-1}(j), j\right) \quad$ and $\quad K_{j}^{\prime \prime}=K_{j}^{\prime \prime \prime}=\left[\pi^{-1}(i), j\right)$.

In particular, all the $K_{r}$-sets above are proper, for $r \in\{i, j\}$.

Proof. In light of Lemma 6.8, the last statement is a consequence of the explicit forms given for the $K$-sets. We need to prove those explicit forms are correct. By Lemma $6.6(2)$ we know that $d \in\{0,1\}$. Assume first that $d=0$ and write $w=s=(i j)$.

The descriptions of the alcoves $\mathbf{v}, \mathbf{v}^{\prime}, \mathbf{v}^{\prime \prime}, \mathbf{v}^{\prime \prime \prime}$ at the end of the previous subsection imply the following formulas, for every $0 \leq k \leq n-1$ and $1 \leq r \leq n$ :

$$
\begin{aligned}
& \mu_{k}(r)=v_{0}(r)+\sum_{l=1}^{k}\left[\mathbf{e}_{\pi(l)}(r)-\mathbf{e}_{l}(r)\right], \\
& \mu_{k}^{\prime}(r)=v_{0}(r)+\sum_{l=1}^{k}\left[\mathbf{e}_{\pi s(l)}(r)-\mathbf{e}_{l}(r)\right], \\
& \mu_{k}^{\prime \prime}(r)=v_{0}(s(r))+\sum_{l=1}^{k}\left[\mathbf{e}_{s \pi(l)}(r)-\mathbf{e}_{l}(r)\right], \\
& \mu_{k}^{\prime \prime \prime}(r)=v_{0}(s(r))+\sum_{l=1}^{k}\left[\mathbf{e}_{s \pi s(l)}(r)-\mathbf{e}_{l}(r)\right],
\end{aligned}
$$

where the sums are interpreted to be 0 if $k=0$. Now we need a definition to simplify the notation.

\section{Definition 6.10.}

$$
\begin{aligned}
& \Delta(s \pi s, \pi, k)(r)=\sum_{l=1}^{k}\left[\mathbf{e}_{s \pi s(l)}(r)-\mathbf{e}_{\pi(l)}(r)\right], \\
& \Delta(s \pi, \pi s, k)(r)=\sum_{l=1}^{k}\left[\mathbf{e}_{s \pi(l)}(r)-\mathbf{e}_{\pi s(l)}(r)\right] .
\end{aligned}
$$

We then deduce the following two formulas:

$$
\begin{aligned}
\mu_{k}^{\prime \prime \prime}(r) & =\mu_{k}(r)+\left[v_{0}(s(r))-v_{0}(r)\right]+\Delta(s \pi s, \pi, k)(r), \\
\mu_{k}^{\prime \prime}(r) & =\mu_{k}^{\prime}(r)+\left[v_{0}(s(r))-v_{0}(r)\right]+\Delta(s \pi, \pi s, k)(r) .
\end{aligned}
$$

There are six possible configurations of the four integers $i, j, i^{\prime}, j^{\prime}$ (e.g. $i<i^{\prime}<$ $\left.j<j^{\prime}\right)$. Although Lemma 6.8 implies that $s\left(i^{\prime}\right)=i^{\prime}$ and $s\left(j^{\prime}\right)=j^{\prime}$ we retain the $s$ in 
the notation in much of what follows, as it clarifies some calculations (in particular the calculation of the values in Table 1). A property that the six configurations of $i^{\prime}, j^{\prime}, i, j$ have in common which we use below is $s\left(i^{\prime}\right)<j^{\prime}$ and $i^{\prime}<s\left(j^{\prime}\right)$. We note that the four intervals $\left[i^{\prime}, s\left(j^{\prime}\right)\right),\left[s\left(i^{\prime}\right), j^{\prime}\right),\left[j^{\prime}, s\left(i^{\prime}\right)\right),\left[s\left(j^{\prime}\right), i^{\prime}\right)$ (which are really only two distinct intervals here) are all proper intervals in $\mathbb{Z} / n \mathbb{Z}$, in the sense of Lemma 6.6 .

There are some divisions into cases that are necessary, namely

- Case(1): $\pi\left(i^{\prime}\right)=i$ and $\pi\left(j^{\prime}\right)=j$,

- Case (2): $\pi\left(i^{\prime}\right)=j$ and $\pi\left(j^{\prime}\right)=i$.

and

- $\operatorname{Case}(\mathrm{A}):\left(v_{0}(i), v_{0}(j)\right)=(0,1)$

- $\operatorname{Case}(\mathrm{B}):\left(v_{0}(i), v_{0}(j)\right)=(1,0)$

- $\operatorname{Case}(\mathrm{C}):\left(v_{0}(i), v_{0}(j)\right)=(0,0)$ or $(1,1)$.

When discussing these cases we will refer to them as Case(A.1), etc. There are a total of six cases, and it is necessary to handle each of them separately. While carrying out this task it is necessary to make frequent reference to the following table giving the values of the functions $\Delta(s \pi s, \pi, k)(r)$ and $\Delta(s \pi, \pi s, k)(r)$ for $r=i, j$.

TABLE 1 . The $\Delta$ functions for $r=i, j$.

\begin{tabular}{|c|c|c|c|c|}
\hline Case $\backslash \Delta$ & $\Delta(s \pi s, \pi, k)(i)$ & $\Delta(s \pi s, \pi, k)(j)$ & $\Delta(s \pi, \pi s, k)(i)$ & $\Delta(s \pi, \pi s, k)(j)$ \\
\hline 1 & $-\xi_{\left[i^{\prime}, s\left(j^{\prime}\right)\right)}(k)$ & $\xi_{\left[s\left(i^{\prime}\right), j^{\prime}\right)}(k)$ & $-\xi_{\left[s\left(i^{\prime}\right), j^{\prime}\right)}(k)$ & $\xi_{\left[i^{\prime}, s\left(j^{\prime}\right)\right)}(k)$ \\
\hline 2 & $\xi_{\left[s\left(i^{\prime}\right), j^{\prime}\right)}(k)$ & $-\xi_{\left[i^{\prime}, s\left(j^{\prime}\right)\right)}(k)$ & $\xi_{\left[i^{\prime}, s\left(j^{\prime}\right)\right)}(k)$ & $-\xi_{\left[s\left(i^{\prime}\right), j^{\prime}\right)}(k)$ \\
\hline
\end{tabular}

The first two columns are computed directly from the definitions. The third and fourth columns can be derived from the first two, using the relations

$$
\begin{aligned}
& \Delta(s \pi, \pi s, k)(i)=-\Delta(s \pi s, \pi, k)(j), \\
& \Delta(s \pi, \pi s, k)(j)=-\Delta(s \pi s, \pi, k)(i) .
\end{aligned}
$$

Now we begin the process of deducing the explicit descriptions of the $K$-sets asserted in this lemma. We have six cases (A.1, A.2, B.1, B.2, C.1, C.2) to consider. We claim that in fact cases A.2 and B.1 do not occur. Take for example Case (A.2). Taking $r=i$ in (5) and looking at the table we get

$$
\mu_{k}^{\prime \prime \prime}(i)=\mu_{k}(i)+1+\xi_{\left[s\left(i^{\prime}\right), j^{\prime}\right)}(k),
$$

from which it follows (since the $\mu$-functions take values in $\{0,1\}$ ) that $\left[s\left(i^{\prime}\right), j^{\prime}\right)=\emptyset$, a contradiction. A similar and equally easy contradiction arises in Case (B.1).

The remaining four cases are A.1, B.2, C.1, and C.2. We will discuss B.2 as an example; the others are similar. Using (5) and (6) and the table we get

$$
\begin{aligned}
\mu_{k}^{\prime \prime \prime}(i) & =\mu_{k}(i)-1+\xi_{\left[s\left(i^{\prime}\right), j^{\prime}\right)}(k), \\
\mu_{k}^{\prime \prime \prime}(j) & =\mu_{k}(j)+1-\xi_{\left[i^{\prime}, s\left(j^{\prime}\right)\right)}(k), \\
\mu_{k}^{\prime \prime}(i) & =\mu_{k}^{\prime}(i)-1+\xi_{\left[i^{\prime}, s\left(j^{\prime}\right)\right)}(k), \\
\mu_{k}^{\prime \prime}(j) & =\mu_{k}^{\prime}(j)+1-\xi_{\left[s\left(i^{\prime}\right), j^{\prime}\right)}(k) .
\end{aligned}
$$


These imply the first four lines of the following relations between the associated sets, the last two being consequences of Lemma 6.6 (3):

$$
\begin{aligned}
K_{i}^{\prime \prime \prime} & =K_{i} \cap\left[s\left(i^{\prime}\right), j^{\prime}\right) & & K_{i}^{c} \cap\left[s\left(i^{\prime}\right), j^{\prime}\right)^{c}=\emptyset, \\
K_{j}^{\prime \prime \prime} & =K_{j} \amalg\left[i^{\prime}, s\left(j^{\prime}\right)\right)^{c}, & & \\
K_{i}^{\prime \prime} & =K_{i}^{\prime} \cap\left[i^{\prime}, s\left(j^{\prime}\right)\right) & & \left(K_{i}^{\prime}\right)^{c} \cap\left[i^{\prime}, s\left(j^{\prime}\right)\right)^{c}=\emptyset, \\
K_{j}^{\prime \prime} & =K_{j}^{\prime} \amalg\left[s\left(i^{\prime}\right), j^{\prime}\right)^{c}, & & \\
{[i, j) } & \subset K_{i}^{c} \cap K_{j} & & {[i, j) \subset\left(K_{i}^{\prime \prime \prime}\right)^{c} \cap K_{j}^{\prime \prime \prime}, } \\
{[i, j) } & \subset\left(K_{i}^{\prime}\right)^{c} \cap K_{j}^{\prime} & & {[i, j) \subset\left(K_{i}^{\prime \prime}\right)^{c} \cap K_{j}^{\prime \prime} . }
\end{aligned}
$$

The next step is to use these properties to deduce the precise descriptions of the sets $K_{i}, K_{j}, K_{i}^{\prime}, K_{j}^{\prime}$, etc. Note that (11), (12), and (15) are consequences only of (7), (8), and Lemma 6.6.

Claim 6.11. The relations (11), (12), and (15) imply that the sets $K_{i}, K_{j}, K_{i}^{\prime \prime \prime}$ and $K_{j}^{\prime \prime \prime}$ are proper.

Proof. It is obvious from (15) that $K_{i} \neq \mathbb{Z} / n \mathbb{Z}$, and $K_{i}^{\prime \prime \prime} \neq \mathbb{Z} / n \mathbb{Z}$. If $K_{i}=\emptyset$, then (11) implies that $\left[s\left(i^{\prime}\right), j^{\prime}\right)^{c}=\emptyset$, a contradiction. Therefore $K_{i}$ is proper. If $K_{i}^{\prime \prime \prime}=\emptyset$, then (11) implies that $K_{i}=\left[j^{\prime}, s\left(i^{\prime}\right)\right)$, and thus by Lemma 6.6 (1), $i=s\left(i^{\prime}\right)$ and $j=i^{\prime}$, contrary to Lemma 6.8. Therefore $K_{i}^{\prime \prime \prime}$ is proper. Similar arguments using only (11), (12), and (15) show that $K_{j}$ and $K_{j}^{\prime \prime \prime}$ are both proper.

Now using the claim and Lemma 6.6 we can describe explicitly the sets $K_{i}, K_{i}^{\prime \prime \prime}$, $K_{j}, K_{j}^{\prime \prime \prime}$. In fact, since $K_{i}$ and $K_{i}^{\prime \prime \prime}$ are both half-open intervals with upper endpoint $i$, we see from $(11)$ that $K_{i}=\left[j^{\prime}, i\right)$ and $K_{i}^{\prime \prime \prime}=\left[s\left(i^{\prime}\right), i\right)$. Moreover, since $K_{j}$ and $K_{j}^{\prime \prime \prime}$ are both intervals with upper endpoint $j$, we see from (12) that $K_{j}=\left[i^{\prime}, j\right.$ ) and $K_{j}^{\prime \prime \prime}=\left[s\left(j^{\prime}\right), j\right)$.

We can now argue similarly to determine the remaining four $K$-sets explicitly. Rather than repeating the arguments, note that we deduced the first four sets formally from (7), (8) and Lemma 6.6. Now (9) and (10) are formally the result of altering (7) and (8) in the following way: in the $\mu$-functions replace "I' with " and "no primes" with ', and replace $i^{\prime}$ with $s\left(i^{\prime}\right)$ and $j^{\prime}$ with $s\left(j^{\prime}\right)$. Applying the same rule to any formal consequence of $(7),(8)$ and Lemma 6.6 gives us a formal consequence of $(9),(10)$ and Lemma 6.6 , so we can simply read off the explicit descriptions of the remaining $K$-sets by applying the rule to the four sets already determined. We have therefore proved the following:

$$
\begin{aligned}
K_{i} & =\left[j^{\prime}, i\right), & K_{i}^{\prime \prime \prime} & =\left[s\left(i^{\prime}\right), i\right), \\
K_{j} & =\left[i^{\prime}, j\right), & K_{j}^{\prime \prime \prime} & =\left[s\left(j^{\prime}\right), j\right), \\
K_{i}^{\prime} & =\left[s\left(j^{\prime}\right), i\right), & K_{i}^{\prime \prime} & =\left[i^{\prime}, i\right), \\
K_{j}^{\prime} & =\left[s\left(i^{\prime}\right), j\right), & K_{j}^{\prime \prime} & =\left[j^{\prime}, j\right) .
\end{aligned}
$$

Now recalling that $s\left(i^{\prime}\right)=i^{\prime}$ and $s\left(j^{\prime}\right)=j^{\prime}$ and that we are in Case (2) yields the desired result. This completes the proof in Case (B.2). The other three cases are similar and will be omitted. This completes the proof of the lemma when $d=0$.

When $d=1$, we proceed as above. We give only a sketch of how the argument differs from that above. Recall the explicit expressions for the four elements $x, x w$, 
$w x$, and $w x w$ given in the previous section, with $d=1$ :

$$
\begin{aligned}
x & =v_{0} \pi, \\
x w & =\left(v_{0}+\left(\mathbf{e}_{\pi(i)}-\mathbf{e}_{\pi(j)}\right)\right) \pi s, \\
w x & =\left(s\left(v_{0}\right)+\left(\mathbf{e}_{i}-\mathbf{e}_{j}\right)\right) s \pi, \\
w x w & =\left(s\left(v_{0}\right)+\left(\mathbf{e}_{i}-\mathbf{e}_{j}\right)+\left(\mathbf{e}_{s \pi(i)}-\mathbf{e}_{s \pi(j)}\right)\right) s \pi s .
\end{aligned}
$$

Here $s=(i j)$ and $w=\left(\mathbf{e}_{i}-\mathbf{e}_{j}\right) s$. Because the translation parts of these four elements are minuscule vectors, we see as in the proof of Lemma 6.8 that $v_{0}(i)=1$, $v_{0}(j)=0, v_{0}(\pi(i))=0$, and $v_{0}(\pi(j))=1$. This means that we are automatically in Case (A), so we have only two cases (A.1 and A.2) to consider here rather than the six cases of the $d=0$ situation. As before we can use the explicit expressions for $x, x w, w x$, and $w x w$ above to write down expressions for the $\mu$-functions, as in equations (1)-(4). Then one can compute the analogs of equations (5) and (6); using the result of Lemma 6.8 and the explicit values for $v_{0}(r)$ and $v_{0}(\pi(r))$ for $r=i, j$ given above, one finds

$$
\begin{aligned}
\mu_{k}^{\prime \prime \prime}(r) & =\mu_{k}(r)+\Delta(s \pi s, \pi, k)(r), \\
\mu_{k}^{\prime \prime}(r) & =\mu_{k}^{\prime}(r)+\Delta(s \pi, \pi s, k)(r),
\end{aligned}
$$

for $r \in\{i, j\}$. This combined with the table is enough information to proceed as before, imitating the steps in the Case (B.2) described above. The only difference is that where recourse to Lemma 6.6 (3) was made in the $d=0$ case, here we use Lemma 6.6 (4) instead.

As a consequence we get:

Corollary 6.12. Let $\mu$ be the minuscule cocharacter $(1,0, \ldots, 0)$ or $(1, \ldots, 1,0)$ of $G l_{n}$, let $x \in \widetilde{W}$, and let $w$ be a reflection. Then $x, x w, w x$, and $w x w$ cannot all be $\mu$-admissible.

Proof. Suppose $\mu=(1,0, \ldots, 0)$. Let $\mathbf{v}$ be the alcove corresponding to $x$. Because $x$ is $\mu$-admissible, its $K$-sets have the following property: $K_{j_{1}} \cap K_{j_{2}}=\emptyset$, for $j_{1} \neq j_{2}$. For suppose $k$ were an element of the intersection. We recall from [10] the notion of size of an alcove: $\operatorname{size}(\mathbf{v})=\mu_{l}(1)+\cdots+\mu_{l}(n)$ (this is independent of the choice of $l$ ). By definition of the $K$-sets, we have $\mu_{k}\left(j_{1}\right)=1$ and $\mu_{k}\left(j_{2}\right)=1$, so that $\operatorname{size}(\mathbf{v}) \geq 2$, contrary to $\operatorname{size}(\mathbf{v})=\operatorname{size}(\mu)=1$ (the equality $\operatorname{size}(\mathbf{v})=\operatorname{size}(\mu)$ holds because $\mathbf{v}$ is $\mu$-admissible, as one sees from the definition of the Bruhat order on $\widetilde{W})$. Now assume that all four elements $x, x w, w x$, and $w x w$ are $\mu$-admissible, so that this comment applies to each of their $K$-sets. Write $w=w_{i, j ; d}$. Using the explicit forms of the $K$-sets given in Lemma 6.9 we see

$$
\left[\pi^{-1}(i), i\right) \cap\left[\pi^{-1}(j), j\right)=\emptyset \text { and }\left[\pi^{-1}(j), i\right) \cap\left[\pi^{-1}(i), j\right)=\emptyset .
$$

But an easy case-by-case analysis of the (twelve) possible configurations of the numbers $i, j, \pi^{-1}(i)$, and $\pi^{-1}(j)$ shows that these intersections cannot simultaneously be empty. This contradiction yields the desired claim.

A similar argument (replacing $\emptyset$ with $\mathbb{Z} / n \mathbb{Z}$ and $\cap$ with $\cup$ ) works for the case $\mu=(1, \ldots, 1,0)$.

Remark 6.13. It is essential for the corollary that we assume $G=G l_{n}$ and $\mu$ as prescribed. The conclusion is false for instance in the case $G=G l_{3}$ and $\mu=(2,0,0)$, the case $G=G l_{4}$ and $\mu=(1,1,0,0)$, and in the case $G=G S p_{4}$ and $\mu=(1,1,0,0)$. 


\section{The Bernstein function for $G l_{n}, \mu=(1,0, \ldots, 0)$}

Here we prove the main application in the paper.

Proposition 7.1. Let $G=G l_{n}$, let $\mu$ be one of the minuscule coweights $(1,0, \ldots, 0)$ or $(1, \ldots, 1,0)$ of $G l_{n}$, and let $\phi=\sum_{w \in \widetilde{W} a_{w} \widetilde{T}_{w}}$ be the element of the Iwahori-Hecke algebra given by

$$
a_{w}= \begin{cases}0 & \text { if } w \text { is not } \mu \text {-admissible } \\ Q^{l(\mu)-l(w)} & \text { if } w \text { is } \mu \text {-admissible. }\end{cases}
$$

Then $\phi=z_{\mu}$.

Proof. Note that $\operatorname{supp}(\phi)$ is contained in the $\mu$-admissible set and $\phi(\mu)=1$ by construction. Therefore by the characterization proved in Theorem 5.8, it suffices to show that $\phi \in Z(\mathcal{H})$. This we do by verifying the three conditions of Lemma 3.1 .

The fact that $\phi$ satisfies the first condition is an immediate consequence of Lemma 4.5.

It is easy to see that $\phi$ satisfies the third condition. This is an immediate consequence of two observations. Let $x \in \widetilde{W}$ and $\tau \in \Omega$ and write $\operatorname{Int}(\tau)(x)=$ $\tau x \tau^{-1}$. Then

(1) $x$ is $\mu$-admissible if and only if $\operatorname{Int}(\tau)(x)$ is $\mu$-admissible.

(2) $l(\operatorname{Int}(\tau)(x))=l(x)$.

The second observation follows from the fact that subgroup $\Omega$ preserves the set $S_{a}$ under conjugation. The first observation is also an easy consequence of the definitions, using this same property of $\Omega$ along with Lemma 4.4 above and the fact that $W \cdot \mu$ is stable under conjugation by $\tau$ (or even any element of $\widetilde{W}$ ), as is easily checked.

It remains to prove that $\phi$ satisfies the second condition of Lemma 3.1. So assume that $l(s x s)=l(x)-2$. Note that in this case $l(x s)=l(s x)$ and $s(s x) s=x s$, so by Lemma 4.5, sx and $x s$ are simultaneously $\mu$-admissible. There are therefore four cases to consider:

(1) $x, x s, s x$, and sxs are not $\mu$-admissible,

(2) sxs is $\mu$-admissible, but the other three are not,

(3) $s x s, x s$, and $s x$ are $\mu$-admissible, but $x$ is not,

(4) $s x s, x s, s x$, and $x$ are all $\mu$-admissible.

Now in case (1), the second condition of Lemma 3.1 is clearly satisfied. Case (2) does not arise, because the fact that sxs is $\mu$-admissible and $s x$ is not implies by Corollary 4.6 (with sxs instead of $x$ ) that $x$ is $\mu$-admissible, a contradiction. In case (3), the conclusion of the second condition of Lemma 3.1 holds, as is easily checked by the definition of $\phi$. Finally, Corollary 6.12 above implies that case (4) does not arise. This completes the proof.

Remark 7.2. After this paper was written, the author learned that J.-L. Waldspurger, in a letter to Rapoport ([13]), had earlier proved that the function $\phi$ defined in Proposition 7.1 lies in $Z(\mathcal{H})$.

\section{Counting $\mu$-ADmissible Elements}

When trying to make explicit computations of the Bernstein function $z_{\mu}$, it is helpful to know the number of $\mu$-admissible elements of $\widetilde{W}$ (see Conjecture 6). In 
this section we present a few formulas for the cardinalities of $\mu$-admissible sets for some cocharacters of the groups $G l_{n}$ and $G S p_{2 n}$. They illustrate the combinatorial complexity of the $\mu$-admissible sets.

We will only consider here $\mu$-admissible sets for some minuscule cocharacters $\mu$. It turns out that in the minuscule case the support of $z_{\mu}$ is precisely the $\mu$ admissible set (comp. Conjecture 6), as is shown in a forthcoming paper by the author [5]. So one can also interpret the formulas below as counting the number of Iwahori double cosets needed to express the Bernstein function $z_{\mu}$ (as a linear combination of characteristic functions of Iwahori double cosets).

For the groups $G l_{n}$ or $G S p_{2 n}$ and the case where $\mu$ is minuscule, one can enumerate the $\mu$-admissible subset of $\widetilde{W}$ by counting instead the more concrete set of minuscule alcoves of given size, thanks to work of Kottwitz and Rapoport (see Theorem 6.4). For the group $G=G S p_{2 n}$ there is a notion of $G$-alcove and a theorem in 10 analogous to Theorem 6.4 , which we now briefly discuss.

Let $\Theta$ denote the automorphism of $\mathbb{R}^{2 n}$ defined by

$$
\Theta:\left(x_{1}, \ldots, x_{2 n}\right) \mapsto\left(-x_{2 n},-x_{2 n-1}, \ldots,-x_{2},-x_{1}\right) .
$$

The Weyl group $W_{n}$ of $S p_{2 n}$ is the group $(\mathbb{Z} / 2 \mathbb{Z})^{n} \rtimes S_{n} \subset S_{2 n}$. Here is a concrete realization of $W_{n}$ : choose a coordinate system $\left(x_{1}, \ldots, x_{n}, y_{n}, \ldots, y_{1}\right)$ for $\mathbb{Z}^{2 n}$. Then $\mathbf{e}_{i} \in(\mathbb{Z} / 2 \mathbb{Z})^{n}$ is the "switch" $x_{i} \longleftrightarrow y_{i}$ and an element $\sigma \in S_{n}$ acts on the vector by simultaneously permuting the $x_{i}$ 's and the $y_{i}$ 's.

The affine Weyl group for $S p_{2 n}$ is the semidirect product of the Weyl group $W_{n}$ and the lattice in $\mathbb{Z}^{2 n}$ consisting of the elements fixed by $\Theta$ :

$$
\left\{\left(x_{1}, \ldots, x_{n},-x_{n}, \ldots,-x_{1}\right) \mid\left(x_{1}, \ldots, x_{n}\right) \in \mathbb{Z}^{n}\right\} .
$$

The extended affine Weyl group $\widetilde{W}(G)$ of $G S p_{2 n}$ is the semidirect product of $W_{n}$ and the lattice of translations consisting of the elements $\left(x_{1}, \ldots, x_{2 n}\right) \in \mathbb{Z}^{2 n}$ such that there exists a $c \in \mathbb{Z}$ such that

$$
c=x_{1}+x_{2 n}=x_{2}+x_{2 n-1}=\cdots=x_{n}+x_{n+1} .
$$

One can define a set of simple affine reflections $S_{a}$ for $G$ analogous to the case of $G l_{n}$, and $\left(W_{a}, S_{a}\right)$ is a Coxeter group; thus there is a length function and a Bruhat order on $W_{a}$ and on $\widetilde{W}(G)$.

A $G$-alcove is a sequence of vectors $v_{0}, \ldots, v_{2 n-1}, v_{2 n}=v_{0}+(1, \ldots, 1)$ of $\mathbb{Z}^{2 n}$ which is an alcove for $G l_{2 n}$ and for which there exists a $d \in \mathbb{Z}$ such that

$$
v_{2 n-i}=\mathbf{d}+\Theta\left(v_{i}\right),
$$

for $1 \leq i \leq 2 n$, where $\mathbf{d}$ denotes the vector $(d, \ldots, d)$. We call the $G$-alcove minuscule if it is minuscule as an alcove for $G l_{2 n}$.

The group $\widetilde{W}(G)$ acts simply transitively on the set of all $G$-alcoves; we identify $\widetilde{W}(G)$ with the set of all $G$-alcoves using as base point the minuscule $G$-alcove $\omega_{0}, \ldots, \omega_{2 n}$.

The following theorem is the analogue for the symplectic group of Theorem 6.4.

Theorem 8.1 (Kottwitz and Rapoport). Let $G=G S p_{2 n}$, and let $\mu$ denote the minuscule vector $\left(1^{n}, 0^{n}\right)$. Suppose $\mathbf{v}$ is a $G$-alcove and $x$ is the corresponding element of $\widetilde{W}(G)$. Then $\mathbf{v}$ is minuscule of size $n$ if and only if $x$ is $\mu$-admissible.

Proof. This is Theorem 4.5 (3) of [10]. 
For a group $G$ and a dominant cocharacter $\mu$, let $N(G, \mu)$ denote the number of $\mu$-admissible elements of $\widetilde{W}(G)$.

Proposition 8.2. Let $n$ be a positive integer. Then

(1) $N\left(G l_{n},(1,0, \ldots, 0)\right)=2^{n}-1$,

(2) $N\left(G l_{n},(1,1,0, \ldots, 0)\right)=3^{n}-2^{n}-n 2^{n-1}$,

(3) $N\left(G S p_{2 n},\left(1^{n}, 0^{n}\right)\right)=\sum_{i=0}^{n} 2^{n-i} n ! / i !$.

Proof. Since the idea is the same for each of these formulas, we present only the proof of the last one as an example. By Theorem 8.1, we need to count the set of minuscule $G$-alcoves v of size $n$.

One can prove that the analogue of Lemma 6.7 holds: For a $G$-alcove $\mathbf{v}, \mathbf{v}$ is minuscule if and only if $v_{0}$ is a minuscule vector and the following condition holds:

(*) For every $i$ with $1 \leq i \leq 2 n$,

$$
v_{0}(i)=0 \Rightarrow \pi^{-1}(i) \leq i \text { and } v_{0}(i)=1 \Rightarrow \pi^{-1}(i) \geq i .
$$

Moreover, a minuscule $G$-alcove $\mathbf{v}$ is of size $n$ if and only if $v_{0}$ is in the $W_{n}$-orbit of $\left(1^{n}, 0^{n}\right)$.

Each $G$-alcove $\mathbf{v}$ is completely determined by the pair $\left(v_{0}, \pi\right)$, where $x=v_{0} \pi \in$ $X_{*}(T) \rtimes W_{n}=\widetilde{W}(G)$ is the element corresponding to $\mathbf{v}$. Therefore we need to count the pairs $\left(v_{0}, \pi\right)$ such that

- $v_{0}$ is of size $n$ (has precisely $n 1$ 's and $n 0$ 's),

- $\pi \in W_{n}$,

- $\left(v_{0}, \pi\right)$ satisfies $(*)$ above.

We count, for each $\pi$, the $v_{0}$ 's such that $\left(v_{0}, \pi\right)$ satisfies the above conditions. The $\pi$ 's are partitioned according to the subset of the $x_{i}$ 's they move. Fix $d: 0 \leq d \leq n$, and a subset $S=\left\{j_{1}<j_{2}<\cdots<j_{d}\right\}$ of $\{1,2, \ldots n\}$. Suppose $\pi$ moves precisely the $x_{j_{l}}$ 's. It may be considered then as an element of $(\mathbb{Z} / 2 \mathbb{Z})^{d} \rtimes S_{d}$ in the obvious way. We may simplify by writing the subscript $l$ in place of $j_{l}$, and write $\pi=\phi \circ \sigma$, where $\phi \in(\mathbb{Z} / 2 \mathbb{Z})^{d}$ and $\sigma \in S_{d}$. These $\pi$ 's which move precisely $S$ may be further partitioned according to the subset $T=\left\{i_{1}<i_{2}<\cdots<i_{k}\right\} \subset\{1,2, \ldots, d\}$ which $\sigma$ moves, where $0 \leq k \leq d, k \neq 1$. For each $T$ there are precisely $a_{k}$ possible $\sigma$ 's, where $a_{k}$ is the number of "derangements" (permutations with no fixed points) on $k$ letters, and each derangement can be paired with $2^{k}$ "switches" $\phi$ such that the product $\phi \sigma$ still moves precisely the set $S$. The number of sets $T$ (inside $S$ ) with cardinality $k$ is $\left(\begin{array}{l}d \\ k\end{array}\right)$. Therefore the number of $\pi$ 's which move precisely the set $S$ is

$$
\sum_{k=0}^{d}\left(\begin{array}{l}
d \\
k
\end{array}\right) 2^{k} a_{k},
$$

(noting that $a_{1}=0$ ). Now it is easy to see that any $\pi$ which fixes precisely the set $S$ can be paired with $2^{n-d}$ possible vectors $v_{0}$ such that $\left(v_{0}, \pi\right)$ satisfies $\left(^{*}\right)$. Indeed, any $v_{0}$ has exactly $n 1$ 's and $n 0$ 's, and moreover for each $i$ we have $\left(v_{0}(i), v_{0}(2 n+1-i)\right)$ is either $(1,0)$ or $(0,1)$. If $i$ is such that $\pi$ moves $x_{i}$, then only one of these possibilites can occur, by $(*)$, but if $\pi$ fixes $x_{i}$, then both can occur, giving a degree of freedom of 2 in the determination of $v_{0}$. Thus the total number of allowed pairs $\left(v_{0}, \pi\right)$ such that $\pi$ fixed precisely $S$ is $2^{n-d}$ times the number above. 
Finally the number of subsets $S$ is $\left(\begin{array}{l}n \\ d\end{array}\right)$, so we see on summing over $d$ that

$$
N\left(G S p_{2 n},\left(1^{n}, 0^{n}\right)\right)=\sum_{d=0}^{n}\left(\begin{array}{l}
n \\
d
\end{array}\right) 2^{n-d} \sum_{k=0}^{d}\left(\begin{array}{l}
d \\
k
\end{array}\right) 2^{k} a_{k} .
$$

The right-hand side can be simplified, using a generating function coming from the power series expansion of $e^{x} /(1-2 x)$ (this was pointed out by Gerd Mersmann), and we obtain

$$
\sum_{i=0}^{n} 2^{n-i} n ! / i !
$$

Remark 8.3. 1. The formula $N\left(G l_{n},(1,0, \ldots, 0)\right)=2^{n}-1$ was known earlier to Rapoport ([12]).

2. Helena Verrill has found a recursive procedure to calculate the numbers $N\left(G l_{n},\left(1^{r}, 0^{n-r}\right)\right)$.

3. Ilan Vardi has pointed out that the formula (3) can be rewritten as

$$
N\left(G S p_{2 n},\left(1^{n}, 0^{n}\right)\right)=\left[2^{n} n ! \sqrt{e}\right],
$$

where $[x]$ denotes the greatest integer less than or equal to $x$.

\section{More examples of Bernstein functions}

In this section we give the coefficients $a_{x}(Q)$ for the expression of $z_{\mu}$ in terms of the normalized basis elements $\tilde{T}_{x}$ (cf. notation in $\S 2$ ):

$$
z_{\mu}=\sum_{x \text { is } \mu \text {-admissible }} a_{x}(Q) \tilde{T}_{x} .
$$

In the examples below, we will only specify $a_{x}(Q)$ for $x$ a $\mu$-admissible element, since all other coefficients are automatically 0.

For each example we give the number $N$ of $\mu$-admissible elements and the length of $\mu$ (however, we will not explicitly describe the $\mu$-admissible sets). We will always let $\tau$ denote the unique element of $\Omega$ such that $\mu \tau^{-1} \in W_{a}$. We use a short-hand notation for elements in the extended affine Weyl group: for example we write $s_{321} \tau$ instead of $s_{3} s_{2} s_{1} \tau$. We use the following system to number simple reflections. In the $G l_{n}$ case, let $s_{i}=(i, i+1) \in S_{n}$ for $1 \leq i \leq n-1$. Let $s_{0}=(1,0, \ldots, 0,-1)(1, n)$ be the simple affine reflection. For $G S p_{2 n}$, let $s_{i}=(i, i+1)(2 n+1-i, 2 n-i) \in W_{n}$ for $1 \leq i \leq n-1$. Let $s_{n}=(n, n+1) \in W_{n}$. Let $s_{0}=(1,0, \ldots, 0,-1)(1,2 n)$ be the simple affine reflection.

9.1. $G l_{n}$. Examples (1) and (2) below are for nonminuscule cocharacters. They were computed directly from the definition. Examples (3) and (4) were deduced from Theorem 5.8 with the help of Lemma 6.6 and Lemma 6.9. We will explain the method for $G l_{4}$ and $\mu=(1,1,0,0)$ below. The case of $G l_{5}$ and $\mu=(1,1,0,0,0)$ follows the same general pattern, but is considerably more complicated. The details of that case will be omitted.

(1) $n=3, \mu=(2,0,0)$. Then $N=19, l(\mu)=4$, and

$$
a_{x}(Q)= \begin{cases}Q^{2}+Q^{4}, & \text { if } l(x)=0 \\ Q^{l(\mu)-l(x)}, & \text { if } l(x)>0\end{cases}
$$


(2) $n=3, \mu=(2,1,0)$. Then $N=25, l(\mu)=4$, and

$$
a_{x}(Q)= \begin{cases}3 Q^{2}+Q^{4}, & \text { if } l(x)=0, \\ Q+Q^{3}, & \text { if } l(x)=1 \\ Q^{l(\mu)-l(x)}, & \text { if } l(x)>1\end{cases}
$$

(3) $n=4, \mu=(1,1,0,0)$. Then $N=33, l(\mu)=4$, and

$$
a_{x}(Q)= \begin{cases}Q^{2}+Q^{4}, & \text { if } l(x)=0 \\ Q^{l(\mu)-l(x)}, & \text { if } l(x)>0\end{cases}
$$

(4) $n=5, \mu=(1,1,0,0,0)$. Then $N=131, l(\mu)=6$, and

$$
a_{x}(Q)= \begin{cases}2 Q^{4}+Q^{6}, & \text { if } l(x)=0 \\ Q^{3}+Q^{5}, & \text { if } l(x)=1 \\ Q^{2}+Q^{4}, & \text { if } x \in S_{1} \\ Q^{4}, & \text { if } x \in S_{2}, \\ Q^{l(\mu)-l(x)}, & \text { if } l(x)>2 .\end{cases}
$$

Here $S_{1}$ and $S_{2}$ are the following subsets of $\mu$-admissible elements of length two:

$$
\begin{aligned}
& S_{1}=\left\{s_{01} \tau, s_{34} \tau, s_{23} \tau, s_{12} \tau, s_{40} \tau\right\} \\
& S_{2}=\left\{s_{43} \tau, s_{41} \tau, s_{42} \tau, s_{21} \tau, s_{31} \tau, s_{32} \tau, s_{20} \tau, s_{30} \tau, s_{10} \tau, s_{04} \tau\right\}
\end{aligned}
$$

9.2. $G S p_{2 n}$. The calculation for $G S p_{4}$ below was done using the definition, jointly with Robert Kottwitz. The calculation for $G S p_{6}$ was done using a method that will appear in a forthcoming paper ([5]).

(1) $n=2, \mu=(1,1,0,0)$. Then $N=13, l(\mu)=3$, and

$$
a_{x}(Q)= \begin{cases}Q+Q^{3}, & \text { if } l(x)=0 \\ Q^{l(\mu)-l(x)}, & \text { if } l(x)>0\end{cases}
$$

(2) $n=3, \mu=(1,1,1,0,0,0)$. Then $N=79, l(\mu)=6$, and

$$
a_{x}(Q)= \begin{cases}Q^{2}+3 Q^{4}+Q^{6}, & \text { if } l(x)=0, \\ 2 Q^{3}+Q^{5}, & \text { if } l(x)=1, \\ Q^{2}+Q^{4}, & \text { if } l(x)=2, \\ Q+Q^{3}, & \text { if } x \in T_{1}, \\ Q^{3}, & \text { if } x \in T_{2}, \\ Q^{l(\mu)-l(x)}, & \text { if } l(x)>3 .\end{cases}
$$

Here $T_{1}$ and $T_{2}$ are the following sets of $\mu$-admissible elements of length three:

$$
\begin{aligned}
& T_{1}=\left\{s_{321} \tau, s_{232} \tau, s_{123} \tau, s_{210} \tau, s_{101} \tau, s_{012} \tau\right\} \\
& T_{2}=\left\{s_{323} \tau, s_{312} \tau, s_{212} \tau, s_{213} \tau, s_{230} \tau, s_{310} \tau, s_{320} \tau, s_{120} \tau, s_{301} \tau, s_{201} \tau, s_{101} \tau\right\} .
\end{aligned}
$$

9.3. Explanation of the case: $G l_{4}, \mu=(1,1,0,0)$. The strategy is the following:

Step 1. Use Lemma 6.6 and Lemma 6.9 to find all elements $x$ for which there exists a simple reflection $s$ such that $x<s x, x s<s x s$ and $s x s$ is $\mu$-admissible. The proof of Proposition 7.1 shows that these elements are such that $a_{x}(Q) \neq Q^{l(\mu)-l(x)}$. Such elements may or may not exist (none exist for example in the situation of Proposition 7.1) but when they exist they lie at the center of the $\mu$-admissible set, 
i.e. the element $\tau$ (the base alcove) plus perhaps a few other elements of small length.

Step 2. Use the answer to Step 1 to write down a candidate function $\phi$ such that its support is in the admissible set, $\phi(\mu)=1$, and which is hoped to be in the center. For the $x$ 's not belonging to a pair $(x, s)$ as above, set $a_{x}(Q)=Q^{l(\mu)-l(x)}$.

Step 3. Use Lemma 3.1 to verify that $\phi$ is in $Z(\mathcal{H})$.

Step 4. By Theorem 5.8 we conclude $\phi=z_{\mu}$ and therefore we have computed $z_{\mu}$.

To carry this out in our concrete case, note that the element $\tau_{1}=(1,0,0,0)(1234)$ $\in \Omega$ acts transitively on the set of simple reflections $s \in S_{a}$, so in order to find all pairs $(x, s)$ that satisfy the condition in Step 1 it suffices to fix $s=s_{1}=(12)$ and find all the $x$ 's that can be paired with this $s$ (and then apply all powers of $\operatorname{Int}\left(\tau_{1}\right)$ to the pairs found). Once $s$ is fixed, the condition that $x<s x, x s<s x s$ are all $\mu$-admissible can be interpreted, using Lemmas 6.6 and 6.9, as conditions on $\pi$, where $x=v_{0} \pi$. In this case it is possible to determine the possible $\pi$ 's very quickly. Namely, $x<s x$ implies by Lemma 6.6 and Lemma 6.9 that

$$
[1,2)=K_{1}^{c} \cap K_{2}=\left[\pi^{-1}(1), 1\right)^{c} \cap\left[\pi^{-1}(2), 2\right) .
$$

On the other hand, Lemma 6.8 implies that $\left\{\pi^{-1}(1), \pi^{-1}(2)\right\}=\{3,4\}$. The only possibility compatible with all this is $\pi^{-1}(1)=3$ and $\pi^{-1}(2)=4$, so that $\pi=$ (13)(24) or $\pi=(1423)$. In either of these cases, Lemma 6.7 shows that the only possible $v_{0}$ is $(1,1,0,0)$. In the first case for $\pi$ we get $x=(1,1,0,0)(13)(24)=\tau$, i.e., the base alcove. The other element is in fact

$$
(1,1,0,0)(1423)=(1,1,0,0)(13)(24)(12)=\tau s ;
$$

therefore the second element does not occur, because $(\tau s) s<\tau s$. Thus we have proved that for $s=(12)$, the only $x$ such that $x<s x, x s<s x s$ are all admissible is $x=\tau$. Conjugating by $\tau_{1}$ produces no new such $x$ 's, so the answer to Step 1 is $\tau$.

Next we follow the recipe in Step 2 and write

$$
\phi=\left(Q^{2}+Q^{4}\right) \tilde{T}_{\tau}+\sum_{x: l(x)>0} Q^{l(\mu)-l(x)} \tilde{T}_{x},
$$

where in the sum on the right-hand side $x$ ranges only over elements in the $\mu$ admissible set. It is easy to verify that $\phi$ is central, using Lemma 3.1 along the lines of the proof of Proposition 7.1. Therefore by Theorem 5.8 we conclude $z_{\mu}=\phi$, as desired.

\section{Compatibility of Bernstein and Satake isomorphisms}

This section has two goals. First we deduce the compatibility of the Satake and Bernstein isomorphisms from work of Lusztig and Kato. Then we compute, rather indirectly, the Bernstein function for an arbitrary dominant cocharacter of $G_{2}$.

10.1. Bernstein and Satake isomorphisms. Let $W$ denote the finite Weyl group of a split $p$-adic group $G$ over a $p$-adic field $F$. Let $q$ denote the size of the residue field of $F$. Let $T$ be a maximal $F$-split torus in $G$. Let $K \subset G(F)$ be a special maximal compact subgroup. Let $I \subset K$ be an Iwahori subgroup which is in 
good position, meaning that we have the decomposition $\bigcup_{w \in W} I w I=K$. Consider the following diagram:

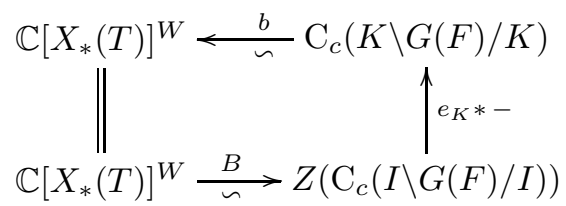

The convolution products in $\mathcal{H}_{K}=C_{c}(K \backslash G(F) / K)$ and $\mathcal{H}=C_{c}(I \backslash G(F) / I)$ are both defined using the Haar measure on $G(F)$ for which $\operatorname{vol}(I)=1$. The right vertical map is convolution by $e_{K}=|K: I|^{-1} \operatorname{char}(K)$. The Satake isomorphism $b$ is defined using the normalizations implicit in 7 and $[8$. Note that this $b$ differs from the usual definition of the Satake isomorphism, which is given for instance in [2]. If we let $b^{\prime}$ denote the Satake isomorphism as defined in [2], then we have the relation $b=|K: I| b^{\prime}$. The difference comes from different choices of Haar measure on $G(F)$ : in $[2$ the measure is chosen such that $K$ has volume 1, whereas implicit in 7 and 8$]$ is the measure giving $I$ volume 1 .

For each dominant cocharacter $\mu \in X_{*}(T)$, define an element

$$
m_{\mu}=\left|W_{\mu}\right|^{-1} \sum_{w \in W} e^{w(\mu)} \in \mathbb{C}\left[X_{*}(T)\right]^{W},
$$

where $W_{\mu}$ denotes the stabilizer of $\mu$ in $W$. By definition the map $B$ sends $m_{\mu}$ to the element $z_{\mu} \in Z(\mathcal{H})$. By Theorem 2.3, $B$ is an algebra isomorphism. We call it the Bernstein isomorphism.

The next proposition seems to be well-known to the experts. It implies that the natural map $e_{K} *-: Z\left(C_{c}(I \backslash G(F) / I)\right) \rightarrow C_{c}(K \backslash G(F) / K)$ is an isomorphism. A proof can be found in a paper of J.-F. Dat (3]). It can also be deduced easily from known results of Lusztig ([7]) and S. Kato ([8]), as is explained below.

Proposition 10.1. The Satake and Bernstein isomorphisms are compatible, i.e., the diagram above is commutative.

Proof. Following Lusztig's notation ([7]), define a function in $\mathcal{H}$ by

$$
\phi_{0}=W(q)^{-1} \sum_{w \in W} T_{w},
$$

where $W(q)=\sum_{w \in W} q^{l(w)}$ is the Poincaré polynomial of $W$. Using $K=\bigcup_{w \in W} I w I$ one sees that $\phi_{0}$ is just another way to write the function $e_{K}$ above. Again following Lusztig, define for each dominant cocharacter $\mu \in X_{d o m}$ the following elements of $\mathcal{H}:$

$$
\begin{aligned}
f_{\mu} & =W(q)^{-1} \sum_{\substack{w \in W \mu W \\
k_{\mu}^{\prime}}} \sum_{\substack{\lambda \preceq \mu \\
\lambda \in \bar{X}_{d o m}}} P_{w_{0} \lambda, w_{0} \mu}(1) z_{\lambda}, \\
c_{\mu}^{\prime} & =q^{-l(\mu) / 2} \sum_{\substack{\lambda \preceq \mu \\
\lambda \in \bar{X}_{d o m}}} P_{w_{0} \lambda, w_{0} \mu}(q) f_{\lambda},
\end{aligned}
$$

where $w_{0}$ is the longest element of $W$ and $P_{x, y}(q)$ are the Kazhdan-Lusztig polynomials for the extended affine Weyl group of $G$. 
Note, $f_{\mu}$ is just another way to write the spherical function $|K: I|^{-1} \operatorname{char}(K \mu K)$, so the functions $f_{\mu}$ form a basis of $\mathcal{H}_{K}$ as $\mu$ ranges over dominant cocharacters (by the Cartan decomposition). Furthermore, since the matrix of Kazhdan-Lusztig polynomials is invertible, Theorem 2.3 implies that $\left\{k_{\mu}^{\prime}, \mu \in X_{d o m}\right\}$ forms a basis for $Z(\mathcal{H})$. To show the commutativity of the diagram it is sufficient to show that $b^{-1} B^{-1}$ and $\phi_{0} *-$ both map $k_{\mu}^{\prime}$ to $c_{\mu}^{\prime}$.

We have $B^{-1}\left(k_{\mu}^{\prime}\right)=\sum_{\lambda \preceq \mu} P_{w_{0} \lambda, w_{0} \mu}(1) m_{\lambda}=\chi_{\mu}$, the character of the module with highest weight $\mu$ (the first equality is trivial, and the second is a theorem of Lusztig ([7])). On the other hand, by a result of Kato (see Theorem 1.8 of [8]) we have

$$
\chi_{\mu}=q^{-l(\mu) / 2} \sum_{\lambda \preceq \mu} P_{w_{0} \lambda, w_{0} \mu}(q) b\left(f_{\lambda}\right) .
$$

It follows that $b^{-1} B^{-1}\left(k_{\mu}^{\prime}\right)=c_{\mu}^{\prime}$. It remains to prove

$$
c_{\mu}^{\prime}=\phi_{0} * k_{\mu}^{\prime}
$$

But this is precisely Proposition 8.6 of [7].

Definition 10.2. We call the function $k_{\mu}=q^{l(\mu) / 2} k_{\mu}^{\prime}$ the test function attached to the dominant cocharacter $\mu$.

10.2. Test functions and Bernstein functions for $G l_{2}$. Let $\mu=(n, m), n \geq m$, be an arbitrary dominant coweight for $G l_{2}$. Let $s_{0}$ denote the unique affine reflection $(1,-1) s_{1}$ where $s_{1}$ the unique reflection in the finite Weyl group $W=\{1,(1,2)\}$.

By the discussion above, $k_{\mu}$ is the element in the Iwahori-Hecke algebra characterized by the following property: $q^{-l(\mu) / 2} k_{\mu}$ is the unique element in the center of the Iwahori-Hecke algebra whose image under $\phi_{0} *-: Z\left(\mathcal{H}_{I}\right) \stackrel{\sim}{\longrightarrow} \mathcal{H}_{K}$ has Satake transform equal to $\chi_{\mu}$, the character of the module with highest weight $\mu$. Here $\phi_{0}$ is the element $W(q)^{-1} \sum_{w \in W} T_{w}$, which in the case of $G l_{2}$ takes the form $(1+q)^{-1}\left(1+T_{s_{1}}\right)$. We write

$$
k_{\mu}=\sum_{x \in \widetilde{W}} k_{\mu}(x) T_{x}
$$

\section{Proposition 10.3.}

$$
k_{\mu}(x)= \begin{cases}0, & \text { if } x \text { in not } \mu \text {-admissible }, \\ 1-q+q^{2}-\cdots+(-q)^{l(\mu)-l(x)}, & \text { if } x \text { is } \mu \text {-admissible. }\end{cases}
$$

Proof (Sketch). Define a function $\tilde{k}_{\mu}$ with coefficients given as in the proposition.

Step 1: Use Lemma 3.1 to show that $\tilde{k}_{\mu}$ is in the center of the Iwahori-Hecke algebra.

Step 2: Write down explicitly the function in the spherical Hecke algebra whose Satake transform is $\chi_{\mu}$. This is

$$
c_{\mu}^{\prime}=q^{-l(\mu) / 2} \sum_{\lambda \preceq \mu} f_{\lambda},
$$

where

$$
f_{\lambda}=(1+q)^{-1} \sum_{x \in W \lambda W} T_{x}
$$


Note that this can be written more explicitly, using that $W \lambda W=\left\{\lambda, s_{1} \lambda, \lambda^{-}, s_{1} \lambda^{-}\right\}$, where $\lambda^{-}$is the antidominant coweight in the $W$-orbit of $\lambda$.

Step 3: Verify by direct computation that $\left(\phi_{0}\right) *\left(q^{-l(\mu) / 2} \tilde{k}_{\mu}\right)=c_{\mu}^{\prime}$.

Conclude: $\tilde{k}_{\mu}=k_{\mu}$.

On the other hand, we have

$$
k_{\mu}=q^{l(\mu) / 2} \sum_{\lambda \preceq \mu} P_{w_{0} \lambda, w_{0} \mu}(1) z_{\lambda}=q^{l(\mu) / 2} \sum_{\lambda \preceq \mu} z_{\lambda},
$$

the second equality holding because the Kazhdan-Lusztig polynomials are trivial for the affine Weyl group of $G l_{2}$. As a result we can invert to calculate the Bernstein functions. We find

$$
q^{l(\mu) / 2} z_{\mu}= \begin{cases}k_{\mu}-q k_{\mu-\check{\alpha}}, & \text { if } \mu \text { is not minuscule, } \\ k_{\mu}, & \text { if } \mu \text { is minuscule. }\end{cases}
$$

Here $\check{\alpha}=(1,-1)$ is the unique simple coroot of $G l_{2}$. Write

$$
z_{\mu}=\sum_{x \in \widetilde{W}} z_{\mu}(x) T_{x}
$$

Using the explicit expression for $k_{\mu}$ given above, we find

\section{Corollary 10.4.}

$$
q^{l(\mu) / 2} z_{\mu}(x)= \begin{cases}0, & \text { if } x \text { is not } \mu \text {-admissible, } \\ 1, & \text { if } l(x)=l(\mu), \\ 1-q, & \text { if } l(x)=l(\mu)-1, \\ 1-2 q+2 q^{2}-\cdots+2(-q)^{r-1}+(-q)^{r}, & \text { if } r=l(\mu)-l(x) \geq 2 .\end{cases}
$$

\section{ACKNOWLEDGEMENTS}

This paper was written during a visit to the Max-Planck-Institut für Mathematik in Bonn. I thank the Institut for the support and for providing an excellent working environment. I am very grateful to Robert Kottwitz and Michael Rapoport for many interesting discussions concerning this material, and for making their results ([10]) available to me before publication.

\section{REFERENCES}

[1] I.N. Bernstein, I.M. Gelfand and S.I. Gelfand, Schubert cells and cohomology of the spaces $G / P$, Uspekhi Mat. Nauk. 28 (1973), 3-26; English translation, Russ. Math. Surv. 28 (1973), 1-26. MR 55:2941

[2] P. Cartier, Representations of p-adic groups: a survey, Proc. of Symp. in Pure Math., vol. 33 (1979), part 1, pp. 111-155. MR 81e:22029

[3] J-F. Dat, Caractères à valeurs dans le centre de Bernstein, J. Reine Angew. Math. 508 (1999), 61-83. MR 2000f:22021

[4] V. Deodhar, On some geometric aspects of Bruhat orderings. I. A finer decomposition of Bruhat cells, Invent. Math. 79, 499-511 (1985). MR 86f:20045

[5] T. Haines, Test Functions for Shimura Varieties: The Drinfeld Case, preprint (1998), to appear in Duke Math. Journal.

[6] J.E. Humphreys, Reflection Groups and Coxeter Groups, Cambridge Studies in Advanced Mathematics, no. 29, Cambridge Univ. Press (1990). MR 92h:20002

[7] G. Lusztig, Singularities, character formulas and a q-analog of weight multiplicities, Astérisque 101-102 (1983), 208-229. MR 85m:17005 
[8] S. Kato, Spherical Functions and a q-Analogue of Kostant's Weight Multiplicity Formula, Inv. Math. 66, 461-468 (1982). MR 84b:22030

[9] R. Kottwitz, Points on some Shimura varieties over finite fields, J. Amer. Math. Soc. 5, (1992), 373-444. MR 93a:11053

[10] R. Kottwitz and M. Rapoport, Minuscule Alcoves for $G l_{n}$ and $G S p_{2 n}$, preprint 1998, to appear in Manuscripta Mathematica.

[11] M. Rapoport, On the Bad Reduction of Shimura Varieties, Automorphic Forms, Shimura Varieties and $L$-functions, part II, Perspectives in Mathematics, vol. 11, Academic Press, San Diego, CA, 1990, 253-321. MR 91g:11065

[12] M. Rapoport, Letter to Waldspurger, January/February 1989.

[13] J.L. Waldspurger, Letter to Rapoport, February 1989.

University of Toronto, Department of Mathematics, 100 St. George Street, Toronto, Ontario, Canada M5S 1A1

E-mail address: haines@math.toronto.edu 